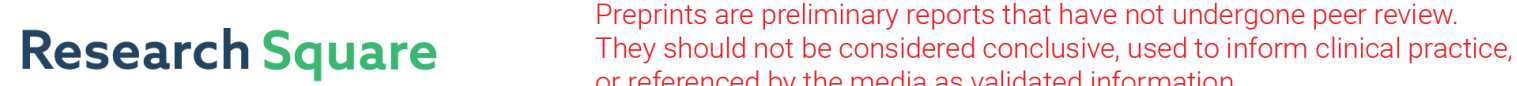 or referenced by the media as validated information. \\ The essence of male scent promotes female puberty and estrus
}

\section{Timothy Holy ( $\nabla$ holy@wustl.edu )}

Washington University in St. Louis https://orcid.org/0000-0002-2429-1071

Xiaoyan Fu

Washington University in St. Louis

Donghoon Lee

Starr Center for Human Genetics, Rockefeller University

\section{Bradley Evans}

Donald Danforth Plant Science Center https://orcid.org/0000-0002-1207-9006

\section{Biological Sciences - Article}

Keywords:

Posted Date: January 20th, 2022

DOI: https://doi.org/10.21203/rs.3.rs-1156787/v1

License: (c) (1) This work is licensed under a Creative Commons Attribution 4.0 International License. Read Full License 


\title{
1 The essence of male scent promotes female puberty and estrus
}

Xiaoyan Fu*, Donghoon Lee, Bradley S Evans \& Timothy E Holy*

\begin{abstract}
Pheromones are chemical signals that trigger a response in another member of the same species. In mice, it has been shown that exposure of females to male pheromones leads to puberty advance and estrus induction ${ }^{1,2,3}$. In particular, these effects can be triggered by conspecific male urine, suggesting that they are related to the chemical composition of this stimulus. Although these phenomena were among the earliest known examples of pheromonal actions, the identities of these chemical signals remain mysterious. Here we identified two small molecules in male urine, termed Calin319 and Calin381 that accounted for much of the vomeronasal neuronal response to male urine and were sufficient and necessary to advance juvenile female puberty and induce female estrus. Besides acting as a primer pheromone, a blend of these two male compounds also acts as a releaser pheromone that resulted in increased investigatory behavior by female mice. These findings demonstrate that Calin319 and Calin381 are crucial male pheromones that regulate female reproductive behavior in mice. This study resolves the long-standing mystery of the molecular code of male urinary chemicals that control female gonadal function.
\end{abstract}

\section{Main}

Social interactions can have a profound impact on neuro-endocrine and reproductive function in mammals. Among the many sensory stimuli provided by conspecifics, olfactory cues are some of the most powerful ${ }^{4,5,6,7,8}$. One of the well-known examples of olfactory modulation of reproductive physiology is the acceleration of juvenile female puberty by male chemosignals, known as the Vandenbergh effect ${ }^{1}$. Another notable example is the Whitten effect ${ }^{2,3}$, a phenomenon in which suppressed ovarian cycling in grouped females is restarted by exposure to male urine. Nearly 50 years after the discovery of these effects, the molecular identity of these chemosignals still remains unknown, with a series of early candidates ${ }^{9,10,11,12,13,14}$ not replicated by subsequent physiology $\mathrm{y}^{6,15,16}$ or behavioral studies ${ }^{17}$. Several major challenges may have prevented identification of these key signals: there are thousands of compounds in mouse urine, behavioral effects are typically generated by compounds of low abundance, and behaviors sometimes depend on (or are synergized by) a blend of compounds making bioassay incompatible with purification ${ }^{8}$. Some of these chemosignals have similar physico-chemical properties that make purification even more challenging 6 . To address this need, we recently developed a new method, ComponentActivity Matching (CAM) $)^{8}$, to select chemical candidates that "explain" patterns of bioactivity across diverse complex biological mixtures. Using this method, we previously identified the first two female mouse sex pheromones ${ }^{8}$. Here we use this approach to solve a long-standing mystery: identifying the essential chemicals of male scent that promote female puberty, estrus, and investigatory behavior.

\section{Identification of male cues for puberty acceleration}


To investigate the olfactory cues that result in puberty acceleration, male urine was applied daily to the external nares of juvenile females with a pipette for four continuous days (Fig. 1a). Consistent with previous results ${ }^{1}$, females treated with male urine entered puberty three days earlier ( $\mathrm{a} \sim 12 \%$ acceleration counting from birth) than those who were treated by water (Fig. 1b). To test a role for any volatiles, we delivered $30 \mu \mathrm{l}$ fresh male urine daily to an inverted perforated petri dish inside mice cages for four continuous days (Fig. 1c). Without being able to touch the male urine, none of the juvenile female mice entered puberty earlier (Fig. 1d). These findings are consistent with previous work showing that puberty acceleration of females requires direct contact and is mediated by one or more non-volatile low molecular weight chemicals ${ }^{17,18,19,20}$. Therefore, we focused on searching for signal chemicals for female puberty acceleration from the non-volatile part of male mice urine.

We first fractionated male urine by size exclusion column into high molecular weight (HMW > $3000 \mathrm{da}$ ) part and low molecular weight (LWM < $3000 \mathrm{da}$ ) part (Fig. 1e). After separately applying them to the external nares of juvenile females with a pipette (Fig. 1f), we found only the LMW urine fraction contained the puberty-accelerating activity (Fig. 1g). To search for chemical signals underlying puberty acceleration, we first fractionated LMW urine extract by high pressure liquid chromatography (HPLC). Then, we performed a forward screen for chemical compounds that drive activity in vomeronasal sensory neurons (VSNs) using a combination of calcium imaging and quantitative liquid chromatography-mass spectrometry (LC-MS) (Fig. 1h). This screen correlated compound abundance across fractions, as measured by negative ion nano LC-MS using high mass resolving power (to enable mass accuracy of a few ppm or four decimal places) (Fig. 2a), against the bioassay. We used calcium imaging, rather than behavior as in some previous studies $^{10,11,12,26}$, for its quantitative readout, high throughput, and possibility of circumventing the frequent dependence of behavior on pheromone blends ${ }^{38}$. Calcium responses were recorded by Objective-Coupled Planar Illumination (OCPI)/light sheet fluorescence microscopy in the intact female mouse vomeronasal epithelium ${ }^{8,16,21}$ from mice expressing GCaMP3 in VSNs. To provide interpretive context, we presented urine extracts from BALB/cJ as well as HPLC urinary fractions matching its endogenous concentration in BALB/cJ male urine $\operatorname{extract}^{8}$ (Fig. 2b-c).

Neurons that responded to either HPLC urinary fractions or whole urine extract fell into 6 clearlydistinguishable groups. Three of the cell groups (clusters 1, 2, and 3) exhibited unambiguous responses to the fractions, in a manner graded with concentration. All three of these fractionresponsive clusters were highly selective for male mouse urine, indicating that the ligand(s) responsible for these neuronal activities only exist or are more concentrated in male mouse urine (Fig. 2c). We performed a preliminary component-activity matching (CAM) analysis of these fractions, asking whether peaks identified by LC-MS were distributed across fractions in a manner that could plausibly explain the activity of these fraction responsive neuronal groups. Among the 20 most-abundant compounds in these fractions, only two likely candidates - as determined from their patterns of concentrations across HPLC fractions -- could explain VSN neuronal response patterns. One candidate with an anion of $\mathrm{m} / \mathrm{z} 319.1760$ (Calin319) (red circle) was primarily from fraction 76, the other one with an anion of $\mathrm{m} / z 381.1919$ (Calin381) (blue circle) primarily from fraction 77 (Fig. 2a, 2d).

We then quantified these compounds in C18 urine extracts of four laboratory strains (BALB/cJ, C57BL/6J, CBA, and DBA). BALB/cJ male urine had the highest abundance of both candidates 
(Extended data Fig. 1a). Therefore, we chose BALB/cJ male urine as a staring material and purified these compounds to homogeneity.

\section{Structural characterization of male ligands}

Based on accurate mass measurement, Calin319 is ionized to a $[\mathrm{M} \mathrm{-} \mathrm{H}]^{-}$with chemical formula of $\mathrm{C}_{15} \mathrm{H}_{27} \mathrm{O}_{7}$, indicating that it is a previously unknown ligand. Following determination of its chemical formula, we solved its structure by using a combination of MS-based methods, including high mass resolving power, tandem MS $\left(\mathrm{MS}^{2}\right.$ and $\left.\mathrm{MS}^{3}\right)$, and hydrogen deuterium exchange (H/DX) to determine the number of hydroxyl groups (Extended data Fig. 2-6). The details of structure elucidation are provided in Methods. Results of these analyses suggested that Calin319 is a glucuronide with a saturated nonyl chain of undetermined isomeric structure.

To test the validity of this structural interpretation, we performed a de novo synthesis of this proposed glucuronide (Extended data Fig. 7a). Stereoselective synthesis, or post-hoc separation of stereoisomeric compounds, can be difficult or impossible, but enzyme catalyzed reactions can avoid this problem and typically produce only one isomer ${ }^{22}$. Therefore, for Calin 319 synthesis, we chose an enzymatic synthetic method. The glucuronidation reaction consists of the transfer of the glucuronosyl group from uridine 5'-diphospho-glucuronic acid (UDPGA) to substrate molecule nonano ${ }^{23}$. Uridine $5^{\prime}$-diphospho (UDP)-glucuronosyltransferase (UGT) is a microsomal glycosyltransferase that can catalyze the transfer of the glucuronic acid component of UDPglucuronic acid to a small hydrophobic molecule, such as straight chain fatty alcohol, to form a unique hydrocarbon glucuronide. However, because purified or synthetic UGT is unavailable, we used BALB/cJ male mice liver microsomes as a resource for UGT. Details of the synthesis method are provided in Methods. In total, we synthesized 16 different glucuronides by using 16 different nonanol isomers (Extended data Fig. 8).

We then tested vomeronasal neuronal responses to these 16 synthetic glucuronide isomers by using light sheet fluorescence microscopy ${ }^{16,21}$. Among these 16 synthetic glucuronides, only 2,6dimethyl-2-heptyl glucuronide showed the same neuronal activity as the endogenous Calin319 (Extended data Fig. 7 b-d). Neuronal responses to the synthetic 2,6-dimethyl-2-heptyl glucuronide and endogenous Calin319 were identical across three different concentration 1:30, 1:300, 1:1000 (Fig. 3a-d). Product-ion mass spectra of the synthetic 2,6-dimethyl-2-heptyl glucuronide and endogenous Calin319 were essentially indistinguishable (Fig. 3e), indicating identical chemical structures of the two. Both synthetic and endogenous compounds also have identical elution time (data not shown). These results conclusively demonstrate that Calin319 is 2,6-dimethyl-2-heptyl glucuronide (Fig. 3e, left inset).

We performed similar analyses for the other male ligand Calin381. The chemical formula of ionized Calin381 [M - H] $]^{-}$is $\mathrm{C}_{20} \mathrm{H}_{29} \mathrm{O}_{7}^{-}$, indicating that Calin381 is another novel ligand. $\mathrm{MS}^{2}$ product ions spectrum of Calin381 contains a series of abundant ions 75, 85, 113 and 175, demonstrating that Calin381 is also a glucuronide (Extended data Fig. 9). After losing a $\mathrm{C}_{5} \mathrm{H}_{4}$ moiety, the structure of the remaining part of Calin381 is indistinguishable to that of Calin319 (Extended data Fig. 2, Extended data Fig. 9). Therefore, the structure of Calin381 includes a hydrocarbon chain, a glucuronic acid and a $\mathrm{C}_{5} \mathrm{H}_{4}$ moiety. Thus it seems likely that Calin381 and 
Calin319 share a common metabolic pathway. A synthetic standard of Calin381 is currently unavailable and was therefore not available for direct testing against endogenous Calin381.

\section{Pheromonal effects of calin319 and calin381}

To further explore the factors that control male ligands Calin319 and Calin381 expression, we quantified urine from BALB/cJ juvenile male mice, BALB/cJ male mice castrated at 21 days of age, and BALB/cJ female mice. Quantitative LC-MS of these samples showed that Calin319 and Calin381 were about 20-fold lower in the urine of 21-day-old juvenile males, and three-fold lessconcentrated in the urine of castrated male mice. Calin319 and Calin381 were not detected in the urine of females (Extended data Fig. 1b). These results indicate that synthesis of Calin319 and Calin381 is affected by gonads in mice.

Given that Calin319 and Calin381 dominate the vomeronasal neuronal responses selective for male mouse urine (Fig. 2c, Fig. 3d), we postulate that they may play an essential role in maletriggered behaviors such as Vandenbergh effect (puberty induction) ${ }^{1}$. To determine whether Calin319 and Calin381 are the male cues for the Vandenbergh effect, we treated singly-housed juvenile female mice with variable chemosensory stimuli by painting them on their external nares with a pipette for four continuous days (Fig. 4a).

Both male urine extract and a blend of Calin319 and Calin381 at endogenous concentrations advance vaginal opening by three days (Fig. 4b). The two compounds have a synergistic effect, with Calin319 having substantial activity on its own but enhanced by the simultaneous presence of Calin381 (Fig. 4c). Moreover, depleting these two compounds from intact male mouse urine (details provided in Methods) extracts eliminates the accelerating property of the urine extract (Fig. 4d), demonstrating that these two compounds are not merely sufficient but also necessary. Castration lowers the amount of Calin319 and Calin381 to one-third of their amount in normal male mice (Extended data Fig. 1b). The diminished Calin319 and Calin381 concentrations in castrated male urine failed to advance juvenile female puberty (Extended data Fig. 1c). Measurements of uterine mass, another often-used indicator of puberty, are consistent with the results measuring vaginal opening time (Fig. 4b, 4e). All of these results indicate that Calin319 and Calin381 play an indispensable role in advancing female puberty.

To determine whether other sensory modalities might contribute to detecting Calin319 and Calin381, we evaluated the response of mutant mice lacking a transient receptor potential channel (TRPC2) that plays a fundamental role in detecting pheromonal and other chemical social recognition signals by the vomeronasal organ $(\mathrm{VNO})^{17,24} \cdot \operatorname{Trp} C 2^{-/-}$female mice were individually treated with water, male ligands or male urine extract. We found that puberty-accelerating properties of these two ligands was dependent on the presence of TRPC2: $\operatorname{Trp} C 2^{--}$juvenile females failed to show puberty acceleration in response to the pheromone(s) in male urine (Fig. 4f), indicating that TRPC 2 function is necessary to detect and respond to pheromones that trigger puberty.

Given their similar nature, the Whitten (ovarian cycling induction) and Vandenburgh (puberty induction) effects ${ }^{1,2,3}$ have been proposed to be mediated by the same urinary pheromones ${ }^{14}$. To determine whether our new discovered male ligands, Calin319 and Calin381, play a vital role in 
the induction of adult female estrus, we directly applied these two compounds to the external nares of group-caged anestrus females (Extended data Fig. 10a). All of these estrous-suppressed females treated by male ligands simultaneously entered estrus cycle after three days' treatment. In contrast, females treated by water failed to start their estrous cycles (Extended data Fig. 10b, c). Thus, male ligands Calin319 and Calin381 not only advance juvenile female puberty, but also induce adult female estrus.

\section{Calin319 and calin381 increase investigatory behavior of female mice}

Social cues in male mouse urine play many important roles in reproduction and social interaction $25,26,27,28,29,30$. Some of these cues elicit endocrine responses such as female puberty induction ${ }^{1,2,3}$, and others can directly release a visible stereotyped behavior such as attraction or avoidance ${ }^{31,32,33,34,35}$. Male mice investigate urine from females significantly longer than urine from males. Two female pheromone compounds, cortigynic acid and corticosteronic acid, caused a similar increased investigatory behavior to that triggered by female urine, when presented at matching concentration ${ }^{8}$. To test if male ligands Calin319 and Calin381 play a role in the investigatory behavior of female mice, we presented samples on a cotton swab and used an optical beam-break detector to measure the duration of investigation periods in freely behaving female mice $^{36,37}$ (Extended data Fig. 11a). We compared investigatory behavior of female mice to a cotton swab with a blend of Calin319 and Calin381 against one with male mice urine extract and water.

Calin319 or Calin381 on its own did not significantly increase the investigation time for females. Females investigated a blend of Calin319 and Calin381 for duration that were more than threefold longer than exhibited for water. A blend of these two male ligands and male urine extract triggered same level investigation time for females (Extended data Fig. 11b, c). This demonstrates that Calin319 and Calin381 play an important role for attracting female investigation in male urine.

Pheromones are among the most widely used signals to organize social behavior and reproduction $^{38}$. In mice, the study of pheromones has impacted not just our understanding of animal behavior but also chemical ecology, sensory transduction, and the circuit and system phenomena that drive behavior. Examples of striking progress on several aggressive, reproductive, and reinforcement behaviors $31,32,33,34,35,39$ have typically started with the discovery of novel olfactory ligands $s^{4,6,8,30,34}$. The ligands have been used as tools to identify the corresponding receptor genes, map circuits, and more deeply probe the behavior through a combination of immediate early gene expression, knockouts and knockins, optogenetics, and circuittracing $^{25,40,41,42}$. While the Vandenberg and Whitten effects ${ }^{1,2,3}$ were among the earliest behaviors known to be induced by male pheromones, they have remained among the most enigmatic. The absence of validated ligands to study processes of estrous induction and early puberty has significantly slowed progress in understanding the neuroendocrine and circuit underpinnings of these behaviors. We believe that discovery of the ligands responsible for these behaviors is another milestone for our understanding of the neuronal control of reproductive behavior in mammals.

\section{Methods}

\section{Mice}


Experiments and animal care were performed in accordance with NIH guidelines and approved by the Washington University in St. Louis Institutional Animal Care and Use Committee (IACUC). Mice were maintained on a 12-h day/night (6:00 to 18:00) cycle in a temperature-controlled facility. Ai38 (GCaMP3)/OMP-Cre male mice aged 8-12 weeks were used for imaging by OCPI microscope ${ }^{16,21}$. Prior to dissection, mice were anesthetized with carbon dioxide. Sexually naive three-month-old female B6D2F1 mice were used for investigatory behavior tests. 22 days old $\mathrm{BALB} / \mathrm{cJ}$ juvenile female mice were used for the puberty acceleration test. All of the mice except for GCaMP3 mice were purchased from Jackson Laboratory.

\section{Solution and stimuli}

In all experiments, vomeronasal neurons were exposed to continuous superfusion with Ringer's $2 \mathrm{mM} \mathrm{MgCl} 2,25 \mathrm{mM} \mathrm{NaHCO} 3,10 \mathrm{mM}$ HEPES and $10 \mathrm{mM}$ glucose. Before each experiment, C18 urine extracts and male ligands were diluted at different concentrations with carboxygenated $\left(95 \% \mathrm{O}_{2}, 5 \% \mathrm{CO}_{2}\right)$ Ringer's solution for the light sheet calcium imaging recordings. Concentrations of male ligands were matched to their original concentration in male urine. Ringer's solution was used for control.

\section{Profiling urine extracts by high resolution mass spectrometry}

LC-MS profiles for C18 urine extracts were acquired with a Thermo LTQ FT interfaced to an Eksigent nanoLC with a custom-packed C18 column (12 cm in length). Each HPLC urinary fraction was run on a $60 \mathrm{~min}$ gradient (from $5 \%$ acetonitrile/95\% $\mathrm{H}_{2} \mathrm{O}$ to $95 \%$ acetonitrile/5\% $\mathrm{H}_{2} \mathrm{O}$ ) at a flow rate of $260 \mathrm{~nL} / \mathrm{min}$. Instrument parameters for LTQ FT were: spray voltage, $1.9 \mathrm{kV}$; capillary voltage, $-32 \mathrm{~V}$; temperature, $275^{\circ} \mathrm{C}$; tube lens, $-222 \mathrm{~V}$.

\section{Light sheet calcium imaging}

For light sheet calcium imaging, $620 \mu \mathrm{m} \times 640 \mu \mathrm{m} \times 300 \mu \mathrm{m}$ imaging volumes were acquired at $0.5 \mathrm{~Hz}$ using a custom OCPI microscope retrofitted with a PCO edge5.5 sCMOS camera) ${ }^{16,21}$. Intact GCaMP3 female vomeronasal epithelia were isolated and adhered onto a nitrocellulose membrane $(0.45 \mu \mathrm{m}$, Millipore Co., MA, USA). Before recording, the VNO was acclimated by superfusing with Ringer's solution for at least $1 \mathrm{~h}$ constantly delivered at a rate of $600 \mu \mathrm{l} / \mathrm{min}$ at $34^{\circ} \mathrm{C}$ by an UltiMate 3000 UPLC (Dionex, Sunnyvale, CA). To measure VSN responses, we applied urine extract and male ligands with different concentrations and used Ringer's solution as control. Stimuli were delivered via the UPLC by an UltiMate 3000 analytical autosampler (Dionex, Synnyvale, CA). Each stimulus was presented once for $5.5 \mathrm{~s}$ in each stimulus block. There were 3 stimulus blocks in total, each presenting the stimulus in different pseudo-randomized order interleaved with the other stimuli. After each stimulation, tissue was superfused with Ringer's solution for $50 \mathrm{~s}$.

Fluorescence intensity of a particular cell in a particular image stack was measured as mean intensity of all pixels within its ROI. Cell responses were measured as $\Delta F / F$ of each stimulus 
period, using a baseline of the corresponding $20 \mathrm{~s}$ pre-stimulus period. For each cell, the reproducibility over all 3 trials was tested by the one-tailed Student's $t$-test against the Ringer's control. Only cells responding to at least one stimulus with $P<0.05$, and $\Delta F / F>0.03$ were counted as responsive cells and neuronal responses were clustered into groups based on patterns of physiological activity. Response clusters were obtained by a variant of the mean shift clustering algorithm $^{43,44}$.

\section{Purification of calin319 and calin381 from male urine}

BALB/cJ male urine from $-80{ }^{\circ} \mathrm{C}$ was thawed, centrifuged and filtered. $\mathrm{C} 18$ urine extract was prepared by methanol extraction, followed by loading to Phenomenex strata-X polymeric reversed-phase, solid-phase extraction (SPE) columns (washing with a 10\% methanol, 2\% acetic acid solution in water and eluting with 100\% methanol). HPLC (Beckman System Gold 126, with 32 Karat software) was performed on a Phenomenex Synergi Max-RP $80 \AA$ (silica based, particle size 4 micron) $250 \times 10 \mathrm{~mm}$ reverse phase column. Purification used a water/methanol gradient, with methanol concentration increasing from 10 to $100 \%$ over $100 \mathrm{~min}$, followed by $100 \%$ methanol for $30 \mathrm{~min}$. HPLC fractions were collected every minute by a Retriever II fraction collector. HPLC fractions inducing male neuronal responses in light sheet calcium imaging were further loaded on a normal phase thin-layer chromatography (TLC) plate and developed at room temperature in a solvent-saturated $\mathrm{N}$-chamber by the ascending technique. Octyl-d-glucuronide methyl ester (Combi-Blocks QB6704) was used as a standard marker for TLC purification. TLC was performed on a glass-support plate with silica gel 60 matrix, particle size of 5-17 $\mu \mathrm{m}$, layer thickness of $250 \mu \mathrm{m}$ (Sigma). The mobile phase system was chloroform $/ \mathrm{methanol} /$ water in ratios of 14:6:1. TLC plate was removed when the solvent line was $2 \mathrm{~cm}$ away from the top of the plate. After a $5 \mathrm{~min}$ wait to allow the solvent to evaporate, purified compounds were recovered by methanol extraction and were further confirmed by LC-MS.

\section{Hydrogen/deuterium exchange (H/DX)}

$\mathrm{H} / \mathrm{DX}$ of Calin319 was initiated by dissolving a dried sample in $\mathrm{D}_{2} \mathrm{O}$ at neutral $\mathrm{pH}$ for about $1 \mathrm{~h}$. Then the same volume of acetonitrile was added prior to conducting ESI MS.

\section{Structural identification of calin319 and calin381}

Chemical structure identification of unknown compound was conducted in the negative ion mode on a Q Exactive and LTQ Orbitrap Velos from Thermo Scientific (West Palm Beach, FL), equipped with the Xcalibur operating system. In the negative ion mode, spray voltage was $3.5 \mathrm{kV}$; capillary voltage and temperature were $32 \mathrm{~V}$ and $275^{\circ} \mathrm{C}$; and Tube lens was $-222 \mathrm{~V}$. Tandem MS $\left(\mathrm{MS}^{\mathrm{n}}\right)$ data was acquired through low-energy CID by using a relative collision energy ranging from 30 to $40 \%$ of the maximum. High resolution mass spectra (HRMS) and H/DX mass spectra were obtained to determine elemental composition and the presence of particular chemical groups. HRMS in the negative-ion mode gave an accurate mass of 319.1760 for molecular ion $[\mathrm{M}-\mathrm{H}]^{-}$, consistent within $1 \mathrm{ppm}$ with the formula $\mathrm{C}_{15} \mathrm{H}_{27} \mathrm{O}_{7}$. This chemical formula is also supported by the isotopic peak distribution, with the $13 \mathrm{C}$ peak being $~ 20 \%$ of the $12 \mathrm{C}$ peak. H/DX MS was performed in negative ion modes, showing that the maximum mass shift after hydrogen/deuterium 
exchange is 3Da in negative mode. This indicates that there are four exchangeable sites (hydroxyl groups) in the molecule, of which one gets ionized in the negative ion mode for detection, suggesting the presence of a carboxylic group (Extended data Fig. 2-3). For further structural elucidation, low-energy negative-ion collision-induced-dissociation (CID) spectra of Calin319 were acquired at both low and high resolving power, allowing elemental composition to be calculated for product ions. For example, one of product ions at $m / z 301.1641\left(\mathrm{C}_{15} \mathrm{H}_{25} \mathrm{O}_{6}{ }^{-}\right)$, formed by the loss of $\mathrm{H}_{2} \mathrm{O}$ from Calin319 $\left(\mathrm{C}_{15} \mathrm{H}_{27} \mathrm{O}_{7}{ }^{-}\right)$. An ion of $m / z 239.1642\left(\mathrm{C}_{14} \mathrm{H}_{23} \mathrm{O}_{5}{ }^{-}\right)$is attributed to the loss of $\mathrm{CO}_{2}$ and $\mathrm{H}_{2} \mathrm{O}$ from $m / z, 301.1641\left(\mathrm{C}_{15} \mathrm{H}_{25} \mathrm{O}_{6}{ }^{-}\right.$) (Extended data Fig. 4). Another example is one of of the product ions at $m / z 259.1539$ is calculated to be $\left(\mathrm{C}_{13} \mathrm{H}_{23} \mathrm{O}_{5}^{-}\right)$, with mass accuracy within 1 ppm, formed by the loss of $\mathrm{C}_{2} \mathrm{H}_{4} \mathrm{O}_{2}$ from Calin319 $\left(\mathrm{C}_{15} \mathrm{H}_{27} \mathrm{O}_{7}{ }^{-}\right)$by cleavage of the carbon next to the carboxylic group (Extended data Fig. 5). The product ions patterns of Calin 319 contains a series of abundant ions $75,85,113$ and 175, consistent with the presence of a glucuronic acid group $^{45,46}$. Product ion $141\left(\mathrm{C}_{9} \mathrm{H}_{17} \mathrm{O}^{-}\right)$indicates that the hydrocarbon chain in Calin319 is a saturated nonyl chain (Extended data Fig. 6).

$\mathrm{MS}^{2}$ product ions spectrum of Calin381 also contains a series of abundant ions 75, 85, 113 and 175, also demonstrating the present of a glucuronic acid group (Extended data Fig. 9). After losing a $\mathrm{C}_{5} \mathrm{H}_{4}$ moiety, the structure of the remaining part of Calin381 is indistinguishable to that of Calin319 (Extended data Fig. 2, Extended data Fig. 9). Therefore, the structure of Calin381 includes a hydrocarbon chain, a glucuronic acid and a $\mathrm{C}_{5} \mathrm{H}_{4}$ moiety (Extended data Fig. 9).

\section{Synthesis of calin319}

We chose an enzymatic synthetic method for Calin319. Because purified or synthetic UGT is unavailable, we used BALB/cJ male mice liver microsomes (Sckisui Xenotech M3000) as a resource for UGT. We incubated $50 \mu \mathrm{lBALB} / \mathrm{cJ}$ male mice liver microsomes M3000, with $8 \mu \mathrm{l}$ alamethicin (Sigma A4665) in $324 \mu 10.1 \mathrm{M}$ potassium phosphate buffer at $4{ }^{\circ} \mathrm{C}$ on ice for $10-15$ min. Then we added $10 \mu \mathrm{l}$ magnesium chloride $(40 \mathrm{mM}), 8 \mu \mathrm{l}$ nonanol. After mixing, tubes were preincubated for $3 \mathrm{~min}$ in a shaking water bath at $37^{\circ} \mathrm{C}$. The reactions were initiated by the addition of $1.292 \mathrm{mg}$ UDP-glucuronic acid (UDPGA, Millipore Sigma U6751) (5 mM). Control incubations were conducted under the same conditions without UDPGA or microsomes. All the incubations were performed in duplicate. Reactions were stopped by the addition of $300 \mu 1$ of icecold acetonitrile. After $30 \mathrm{~min}$, samples were vortex-mixed. Denatured proteins were separated by centrifugation at $4300 \mathrm{rpm}$ and $4{ }^{\circ} \mathrm{C}$ for $10 \mathrm{~min}$ in a Sorvall model T21 super centrifuge (Newton, CT). An aliquot of the supernatant was analyzed by LC-MS. In total, we synthesized 16 different glucuronides by using 16 different nonanol isomers (Extended data Fig. 8).

\section{Removal of calin319 and calin381 from urine extract}

We fractionated $100 \mathrm{~mL}$ of BALB/cJ male mouse urine $\mathrm{C} 18$ extract by HPLC on a water/methanol gradient. LC-MS analysis indicated that two HPLC fractions (out of 100) contained the majority of Calin319 and Calin381, and that relatively few additional compounds co-eluted in these fractions. Moreover, none of co-eluted compounds were among CAM candidate ligands. To approximately reconstruct male urine extract without Calin319 and Calin381, an equal-volume aliquot was taken from each of the remaining 98 fractions; the solvent was removed by evaporation until dryness, and water added to reconstitute the corresponding original volume. 


\section{Puberty acceleration and induction bioassay}

We treated singly-housed juvenile female mice with variable chemosensory stimuli by painting them on their external nares with a pipette for four continuous days. Vaginal openings were checked daily. Male urine was collected from mice of the BALB/cJ strain. To apply the stimulus, mice were restrained by hand in a supine position and slowly stroked with the stimulus until it bubbled at the nostrils. Each female was treated daily with $10 \mathrm{ul}$ stimuli. On day six, at the age of 27 , mice using for measuring uterus were euthanized and body weight was recorded. Mice using for observation of the vaginal openings were checked daily till at the age of 29 or 30 . Each uterus was rapidly dissected, removed of fat, and weighed.

To induce the anestrus stage of the BALB/cJ females, eight six-week-old female mice were cohabituated in a cage for 28 days. After anestrus was verified by examining their vaginal openings, these grouped animals were treated with various olfactory cues. Each female was treated daily with $10 \mathrm{ul}$ stimuli by painting them on their external nares with a pipette for three continuous days. Each day, prior to treatment (between 8:00 and 9:00), all females were examined for their vaginal openings.

\section{Investigation assays}

Female mouse chemosensory investigation was recorded as previously described ${ }^{8,36,37}$. Briefly, eight-week-old B6D2F1 female (Jackson Laboratory) were singly housed from 6 weeks old and kept in 12-h day/night (6:00 to 19:00) cycle. A day's test consisted of 15 min of acclimatization followed by three $120 \mathrm{~s}$ trials (for 3 testing stimuli), using a 20 min gap between trials. Each trial was a presentation of a cotton swab with $20 \mu \mathrm{l}$ stimuli solution. Swabs, mounted in a custom beambreak sensor, were introduced through a hole in the lid of the chamber at the beginning of the recording. Stimulus order was randomized across mice, and the experimenter was blinded to their identity. Periods in which the swab was investigated were extracted algorithmically from the voltage signal from an optical beam-break detector (Extended data Fig. 11a). To exclude false triggers, bouts of investigation lasting less than $15 \mathrm{~ms}$ were discarded. Significance was assessed by paired $t$-test.

\section{Statistical analysis}

Uterine weights were first analyzed by one-way ANOVA, and then the mean between each pairwise combination of groups was compared by using Tukey-Kramer post hoc test. Duration of investigation periods in freely behaving female for different stimuli was assessed by a paired $t$-test.

\section{References}

1. Vandenbergh, J. G. Male odor accelerates female sexual maturation in mice. Endocrinol. 84, 

658-660 (1969).

2. Whitten, W.K. Modification of the oestrous cycle of the mouse by external stimuli associated with the male. J. Endocrinol. 13, 399-404 (1956).

3. Whitten, W.K. Effect of exteroceptive factors on the oestrous cycle of mice. Nature 180, 1436 (1957).

4. Kimoto, H., Haga, S., Sato, K. \& Touhara, K. Sex-specific peptides from exocrine glands stimulate mouse vomeronasal sensory neurons. Nature 437, 898-901 (2005).

5. Haga, S. et al. Integrated action of pheromone signals in promoting courtship behavior in male mice. Elife 3, e0302 (2014).

6. Nodari, F. et al. Sulfated steroids as natural ligands of mouse pheromone-sensing neurons. The Journal of neurosci. 2, 6407-6418 (2008).

7. Stowers, L., Holy, T., Meister, M., Dulac, C. \& Koentges, G. Loss of sex discrimination and male-male aggression in mice deficient for TRP2. Science 295, 1493-1500 (2002).

8. Fu, X. et al. A Molecular Code for Identity in the Vomeronasal System. Cell 163, 311-323 (2015).

9. Nishimura, K., Utsumi, K., Yuhara, M., Fujitani, Y. \& Iritani, A. Identification of pubertyaccelerating pheromones in male mouse urine. J. Exp. Zool. 251, 300-305 (1989).

10. Novotny, M., Ma, W., Wiesler, D. \& Zidek, L. Positive identification of the pubertyaccelerating pheromone of the house mouse: the volatile ligands associating with the major urinary protein. Proc. R Soc. Lond. B Biol. Sci. 266, 2017-2022 (1999).

11. Jemiolo, B., Harvey, S. \& Novotny, M. Promotion of the Whitten effect in female mice by synthetic analogs of male urinary constituents. Proc. Natl. Acad. Sci. 83, 4576-4579 (1986).

12. Jemiolo, B., Andreolini, F., Xie, T., Wiesler, D. \& Novotny, M. Puberty-affecting synthetic analogs of urinary chemosignals in the house mouse, Mus domesticus. Physiol. Behav. 46, 293-298 (1989).

13. Leinders-Zufall, T. et al. Ultrasensitive pheromone detection by mammalian vomeronasal neurons. Nature 405, 792-796 (2000).

14. Schwende, F., Wiesler, D., Jorgenson, J., Carmack, M. \& Novotny, M. Urinary volatile constituents of the house mouse Mus musculus and their endocrine dependency. J. Chem. Ecol. 12, 277-296 (1986).

15. Luo, M., Fee, M., \& Katz, L. Encoding pheromonal signals in the accessory olfactory bulb of behaving mice. Science 299, 1196-1201 (2003)

16. Turaga, D. \& Holy, T. Organization of vomeronasal sensory coding revealed by fast volumetric calcium imaging. J. Neurosci. 32, 1612-1621 (2012).

17. Flanagan, K., Webb, W. \& Stowers, L. Analysis of male pheromones that accelerate female reproductive organ development. PLoS One 6, e16660 (2011).

18. Vandenbergh, J., Finlayson, J., Dobrogosz, W., Dills, S. \& Kost, T. Chromatographic Separation of Puberty Accelerating Pheromone from Male Mouse Urine. Biol. Reprod. 157, 160-265 (1976).

19. Drickamer, L. \& Assmann, S. Acceleration and delay of puberty in female housemice: methods of delivery of the urinary stimulus. Dev. Psychobiol. 14, 487-497(1981).

20. Price, M. \& Vandenbergh, J. Analysis of puberty-accelerating pheromones. J. Exp. Zool. 264, 42-45 (1992).

21. Holekamp, T., Turaga, D. \& Holy, T. Fast three-dimensional fluorescence imaging of activity in neural populations by objective-coupled planar illumination microscopy. Neuron 57, 661$672(2008)$. 
22. Bock, K. \& Köhle, C. UDP-glucuronosyltransferase 1A6: structural, functional, and regulatory aspect. Meth. in Enzymol. 400, 57-75 (2005).

23. Wu, B., Kulkarni, K., Basu, S., Zhang, S. \& Hu, M. First-Pass Metabolism via UDPGlucuronosyltransferase: a Barrier to Oral Bioavailability of Phenolics. J. Pharm. Sci. 100, 3655-3681 (2011).

24. Leypold, B. et al. Altered sexual and social behaviors in trp2 mutant mice. Proc. Natl. Acad. Sci. 99, 6376-6381 (2002).

25. Demir, E. et al. The pheromone darcin drives a circuit for innate and reinforced behaviours. Nature 578, 137-141(2020).

26. Chamero, P. et al. Identification of protein pheromones that promote aggressive behavior. Nature 450, 899-902 (2007).

27. Leinders-Zufall, T. et al. MHC class I peptides as chemosensory signals in the vomeronasal organ. Science 306, 1033-1037 (2004).

28. Lin, D., Zhang, S., Block, E. \& Katz, L. Encoding social signals in the mouse main olfactory bulb. Nature 434, 470-477 (2005).

29. Roberts, S. et al. Darcin: a male pheromone that stimulates female memory and sexual attraction to an Individual male's odour. BMC Bio. 8, 75 (2010).

30. Doyle, W. et al. Faecal bile acids are natural ligands of the mouse accessory olfactory system. Nat. Commun. 7, 11936 (2016).

31. Haga, S. et al. The male mouse pheromone ESP1 enhances female sexual receptive behaviour through a specific vomeronasal receptor. Nature 466, 118-122 (2010).

32. Roberts, S., Davidson, A., McLean, 1., Beynon, R. \& Hurst, J. Pheromonal induction of spatial learning in mice. Science 338, 1462-1465 (2012)

33. Yang, C. et al. Sexually dimorphic neurons in the ventromedial hypothalamus govern mating in both sexes and aggression in males. Cell 153, 896-909 (2013).

34. Ferrero, D. et al. A juvenile mouse pheromone inhibits sexual behaviour through the vomeronasal system. Nature 502, 368-371 (2013).

35. Hattori, T. et al. Self-exposure to the male pheromone ESP1 enhances male aggressiveness in mice. Curr. Biol. 26, 1229-1234 (2016).

36. Holy, T. \& Guo, Z. Ultrasonic songs of male mice. PLoS bio. 3, e386 (2005)

37. Guo, Z. \& Holy, T. Sex selectivity of mouse ultrasonic songs. Chem. Senses 32, 463-473 (2007).

38. Wyatt, T. PHEROMONES AND ANIMAL BEHAVIOUR. Cambridge University Press, Cambridge, UK 2003.

39. Hattori, T. et al. Exocrine gland-secreting peptide 1 is a key chemosensory signal responsible for the Bruce effect in mice. Curr. Bio. 27, 3197-3201(2017).

40. Ishii, K. et al. A labeled-line neural circuit for pheromone-mediated sexual behaviors in mice. Neuron 95, 123-137 (2017).

41. McCarthy, E. et al. A comparison of the effects of male pheromone priming and optogenetic inhibition of accessory olfactory bulb forebrain inputs on the sexual behavior of estrous female mice. Horm. Behav. 89, 104-112 (2017).

42. McCarthy, E. et al. DREADD-induced silencing of the medial amygdala reduces the preference for male pheromones and the expression of lordosis in estrous female mice. Eur. J. Neurosci. 46, 2035-2046 (2017).

43. Comaniciu, D. \& Meer, P. Mean shift: A robust approach toward feature space analysis. Leee T Pattern Anal. 24, 603-619 (2002). 
44. Fukunaga., K. \& Hostetler, L. Estimation of Gradient of a Density-Function, with Applications in Pattern-Recognition. Leee T Info Theory 21, 32-40 (1975).

45. Trontel, J. Quantification of Glucuronide Metabolites in Biological Matrices by LC-MS/MS. Tandem Mass Spec. - Appl. and Prin. 531-558 (2012).

46. Qu, J., Wang, Y., Luo, G. \& Wu, Z. Identification and determination of glucuronides and their aglycones in Erigeron breviscapus by liquid chromatography-tandem mass spectrometry. $J$. Chrom. A. 928,155-62 (2001).

\section{Acknowledgements}

We thank K. Janiszak and J. Romero for their assistance for behavior experiment, H. Schoknecht for mouse care and core facilities at Donald Danforth Plant Science Center. We are grateful to Adam Kepecs for his insightful comments on our manuscript. We also thank Shelei Pan for suggestions and comments. This work was supported by NIH/NIDCD R01 DC005964 and DC010381.

\section{Author information}

Affiliations

Department of Neuroscience, Washington University in St. Louis, St. Louis, MO 63110, USA

Xiaoyan Fu \& Timothy E. Holy

Starr Center for Human Genetics, Rockefeller University, New York, NY 100065, USA

Donghoon Lee

\section{Donald Danforth Plant Science Center, St. Louis, MO 63132, USA}

Bradley S. Evans

Contributions

X.F. and T.E.H. discussed the design of experiments and the results, and wrote the manuscript. X.F. performed all of the experiments and analysis. D.L. helped with calcium imaging and analysis of data. B.E. performed LC-MS profiling. T.E.H. performed the CAM analysis.

Corresponding authors

Xiaoyan Fu and Timothy E Holy

\section{Ethics declarations}


508 Competing interests

509 The authors declare no competing interests.

510

511

512

513

514 


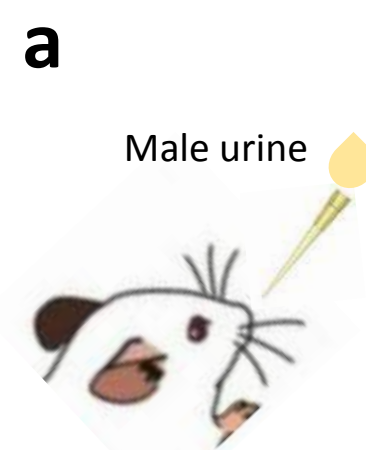

Juvenile female

b

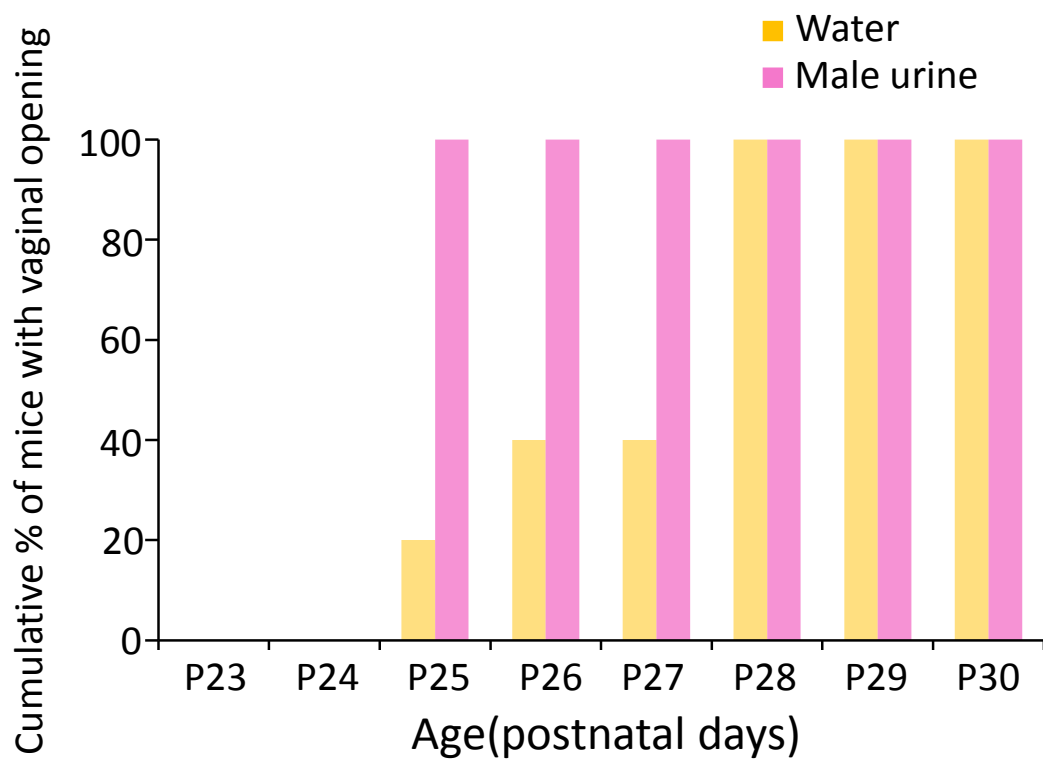

e

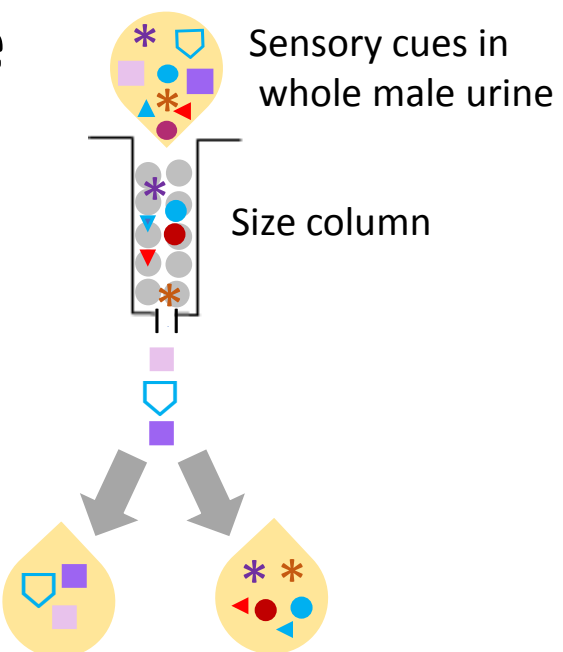

HMW urine fraction LMW urine fraction

h

C18 male urine LMW extract

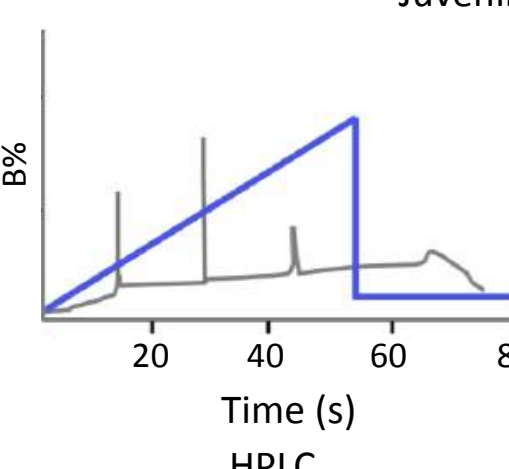

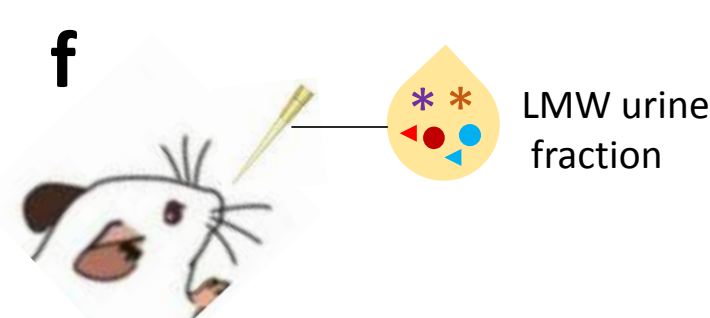

Juvenile female

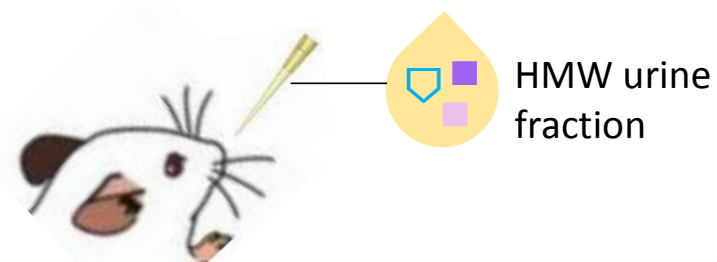

uvenile female d

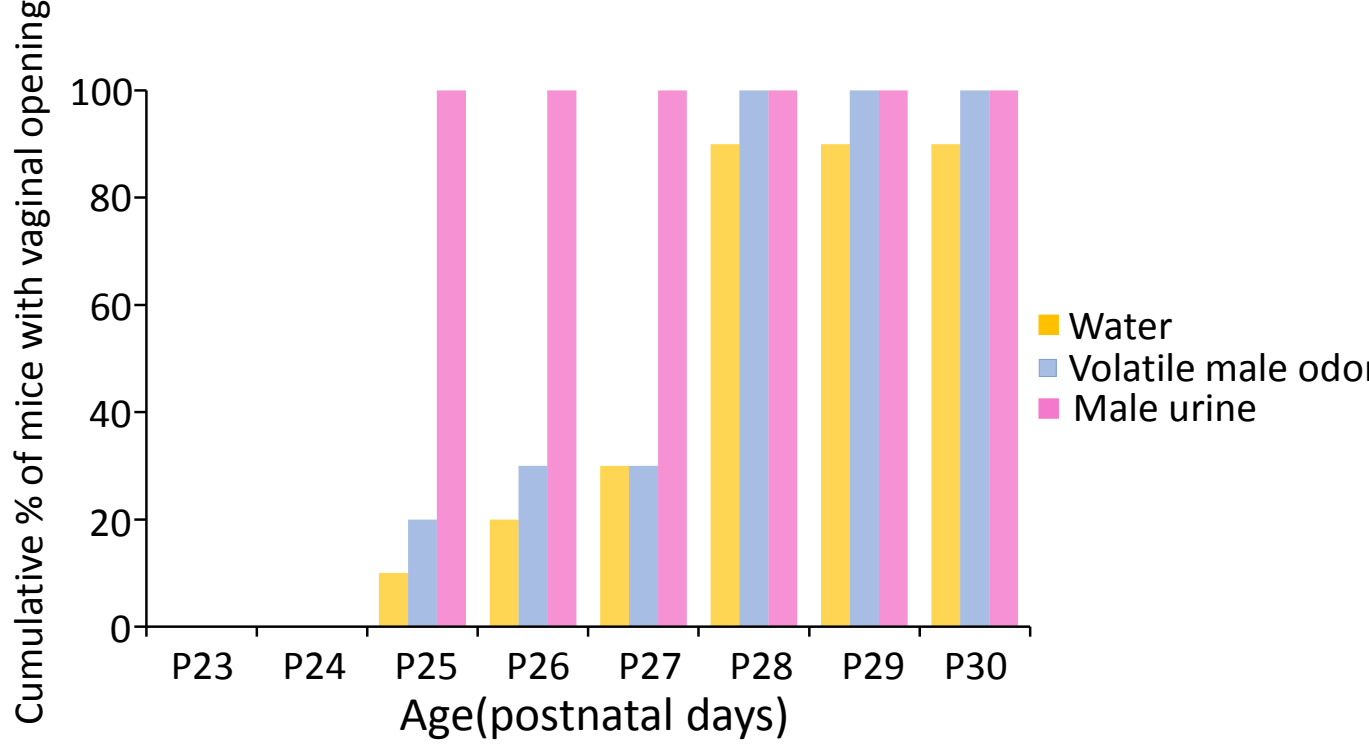

\section{g}

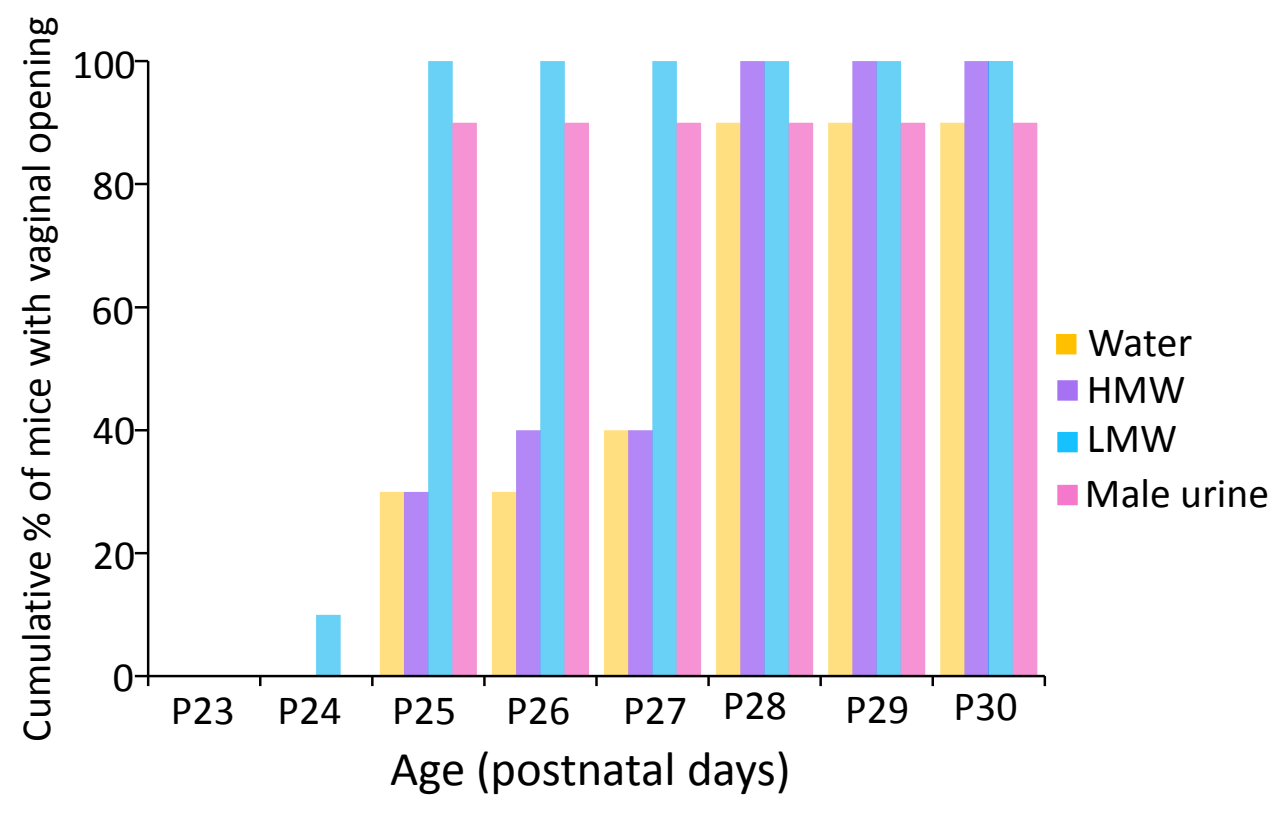

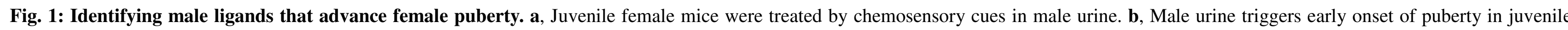

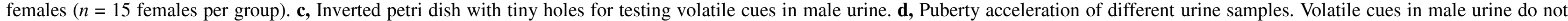

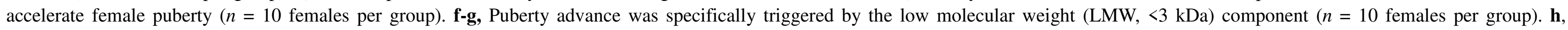
Workflow for purifying and identifying male ligands that activate vomeronasal sensory neurons. 


\section{a}

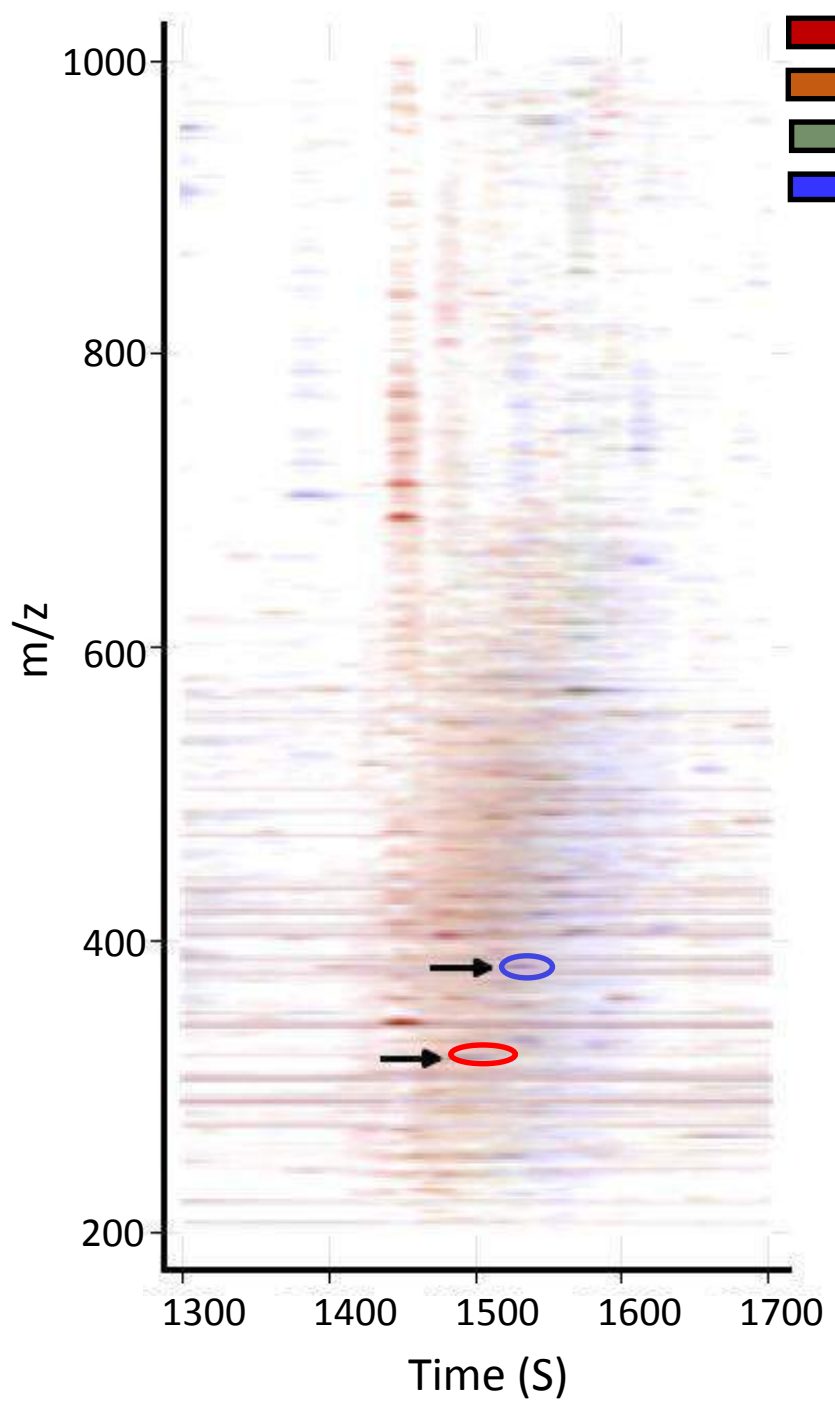

d

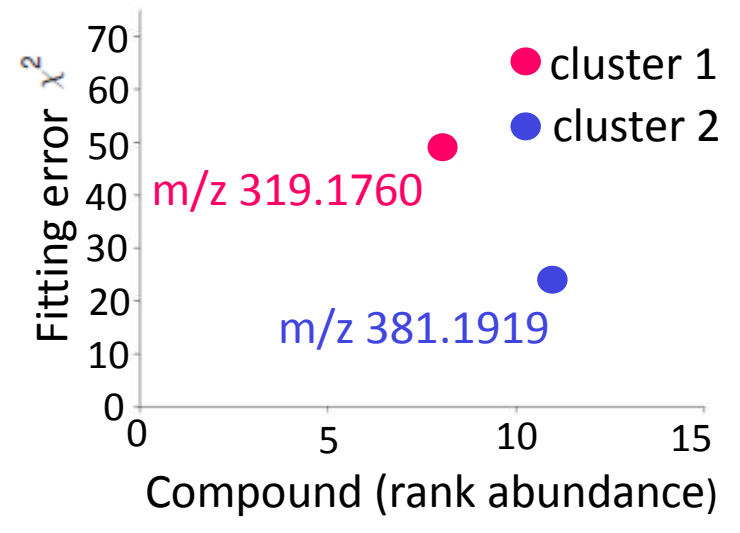

b

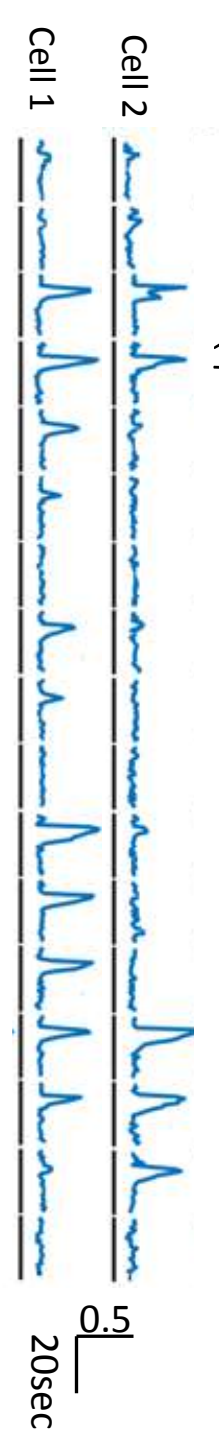

\section{C}

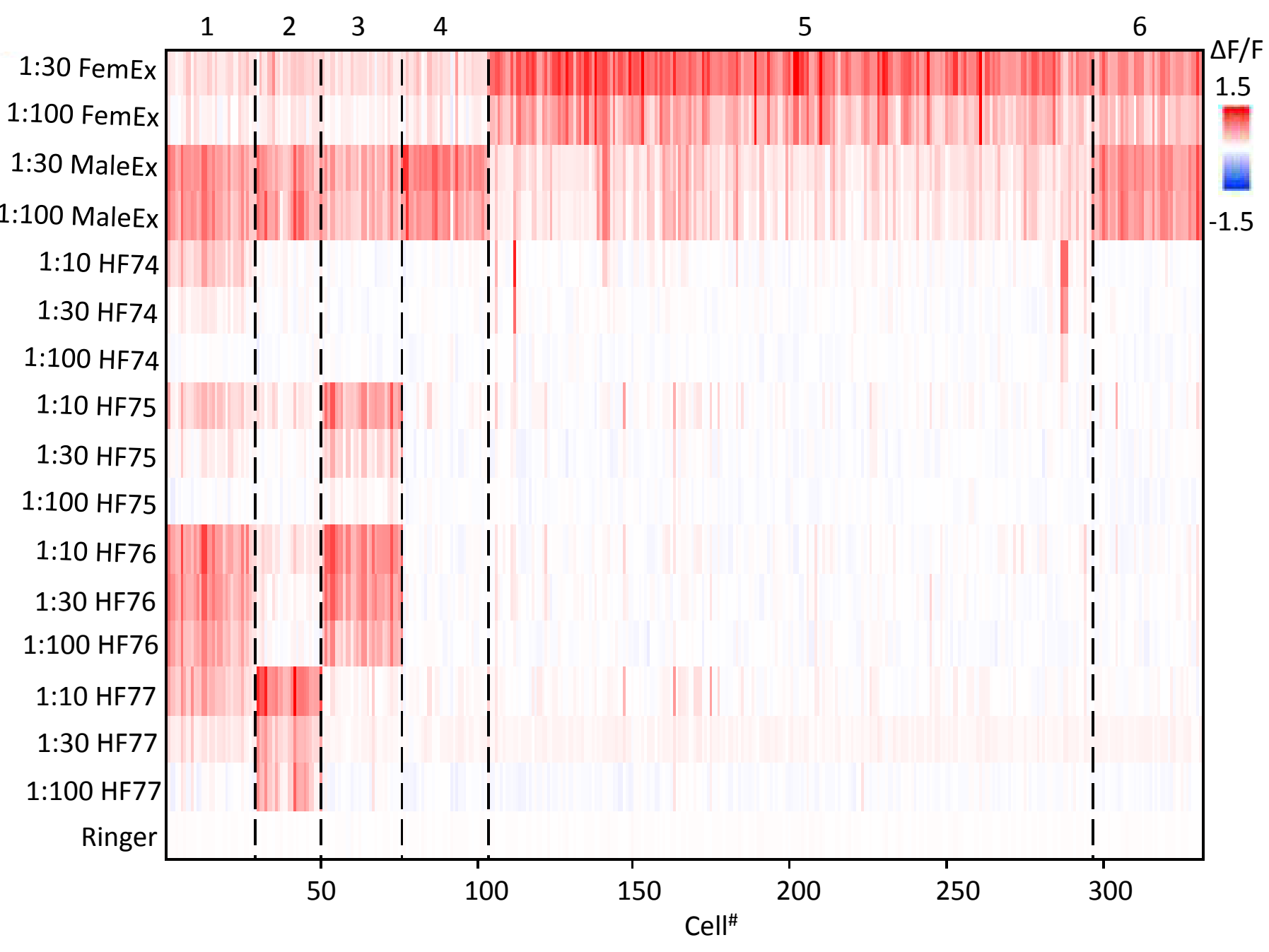

Fig. 2: Identification of male ligand candidates by Component-activity Matching (CAM). a, Distribution of components across HPLC fractions 74-77. Ion count as a function of elution time (high-resolution nanoLCMS/MS) from the column (horizontal axis, elution time marked as seconds) and $m / z$ (vertical axis). (See $\mathrm{d}$ for the information of red and blue circles.) b, Example calcium traces (normalized $\Delta F$ ) from two cells. Amplitude is normalized by the maximum amplitude of all cells recorded. Stimuli are listed at the top. c, Neuronal responses to urine extracts and male ligands. Cells are on columns, and stimuli are on rows. Each cell is represented as a single column, and cells with similar response patterns were grouped. The first three groups correspond to male-selective responses. The color bar indicates normalized response. Cluster identities are reported at the top. $\mathbf{d}$, Abundance vs. neuronal-response explanatory power (as measured by fitting error $\chi^{2}$ ) of ligands in fractions 74-77. Only two peaks (indicated in a, $\mathrm{m} / z 319.1760$ (red) and $\mathrm{m} / \mathrm{z} 381.1919$ (blue)) exhibited fitting errors less than 75 (Fu et al, 2015). 
a
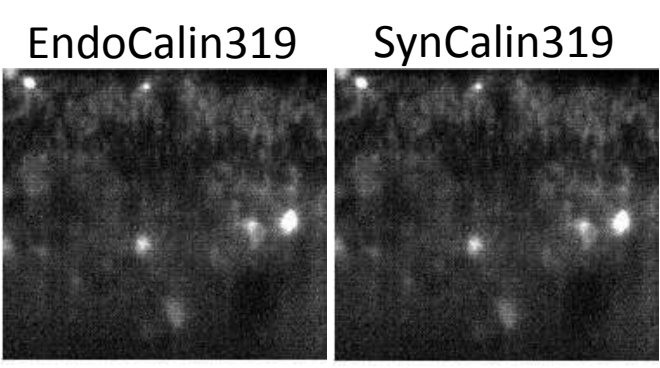

C

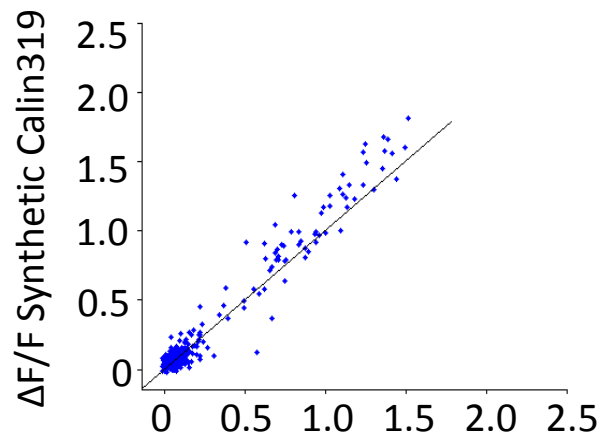

$\Delta F / F$ Endogenous Calin 319

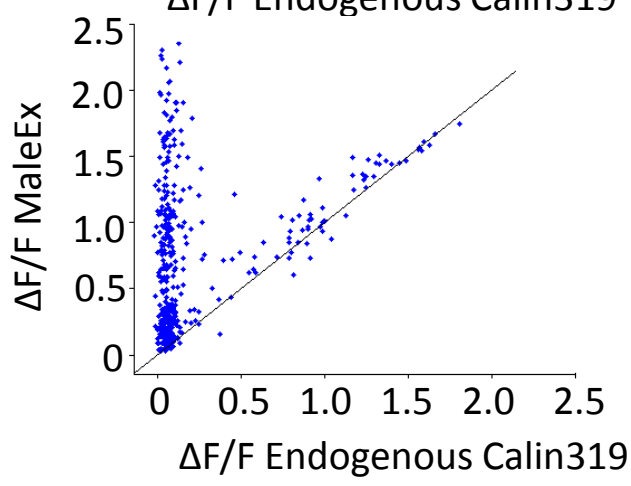

e

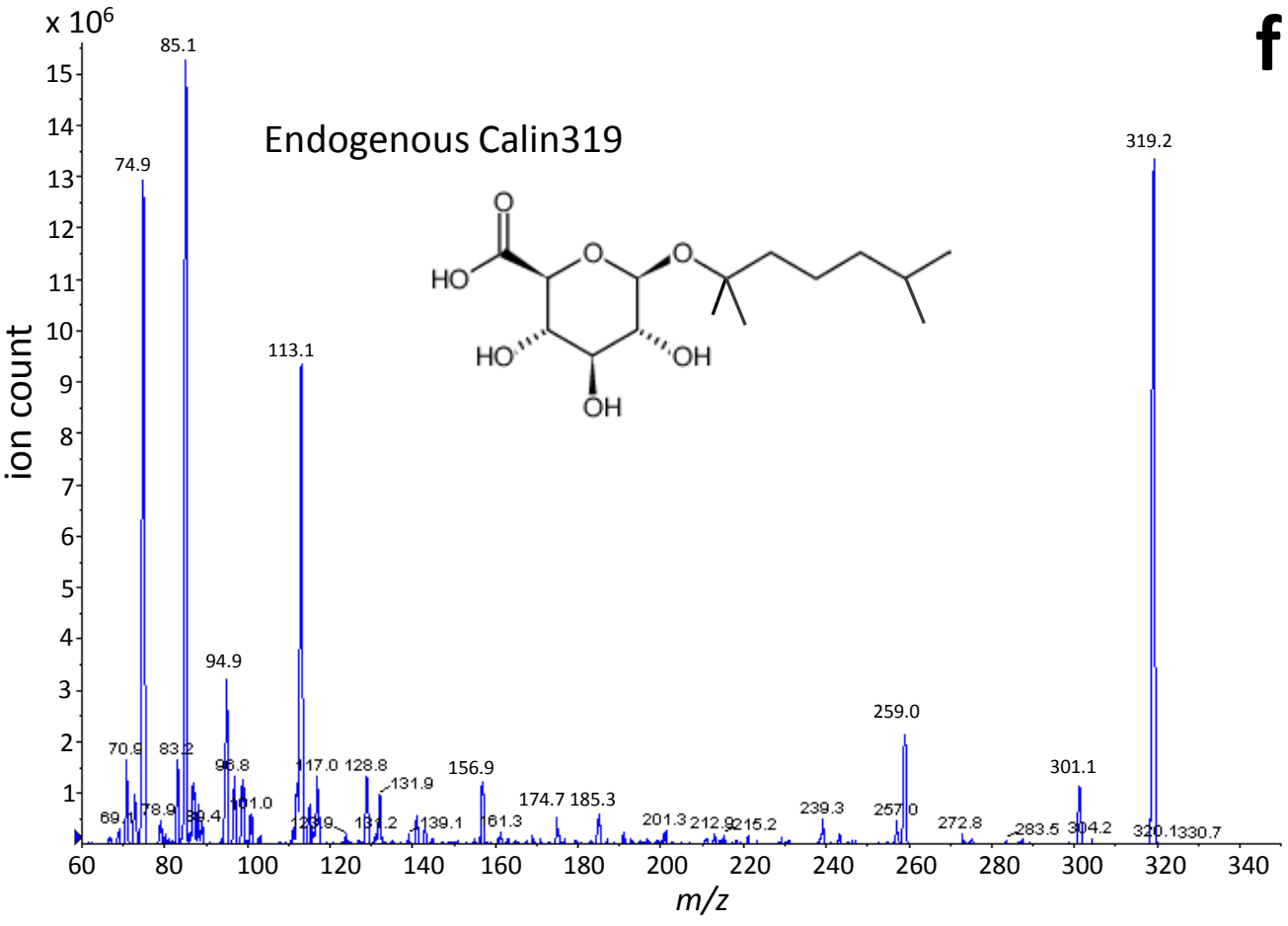

MaleEx

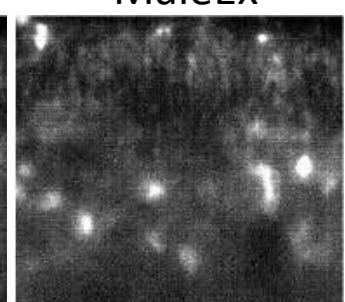

Ringer

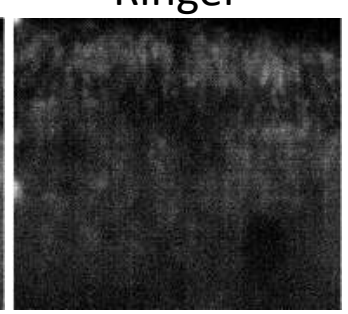

d

1:100 MaleEx

1:1000 MaleEx

1:30 EndoCalin319

1:100 EndoCalin319

1:1000 EndoCalin319

1:30 SynCalin319

1:100 SynCalin319

1:1000 SynCalin319

Ringer b

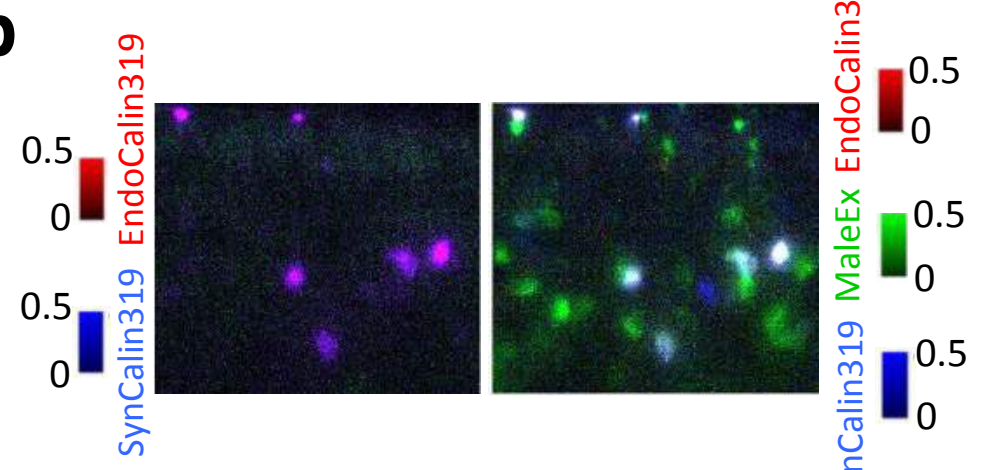

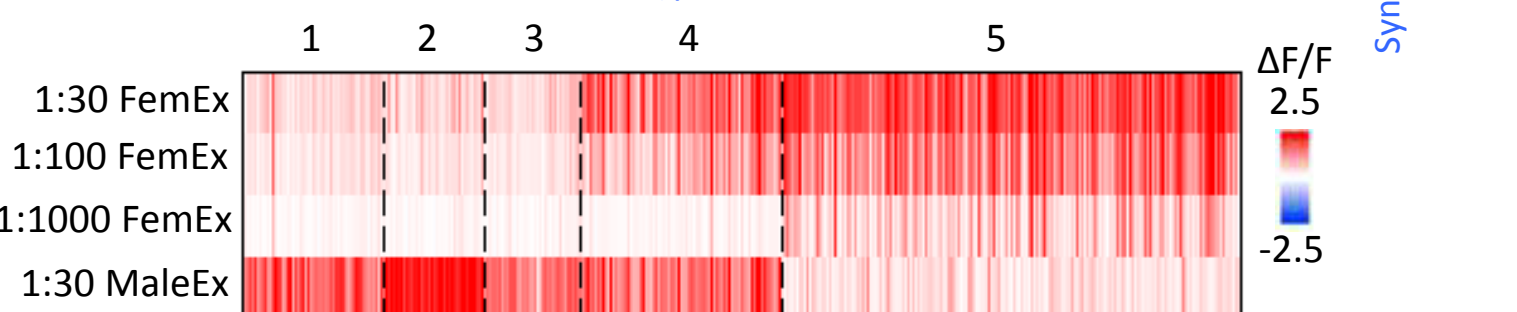

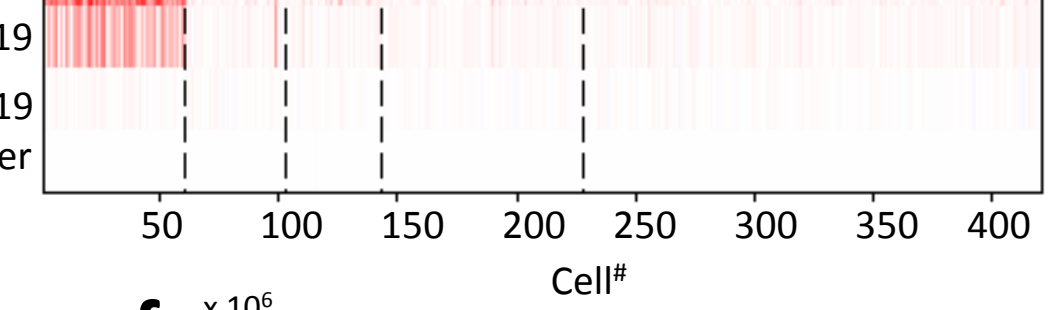

f $\times 10^{6}$

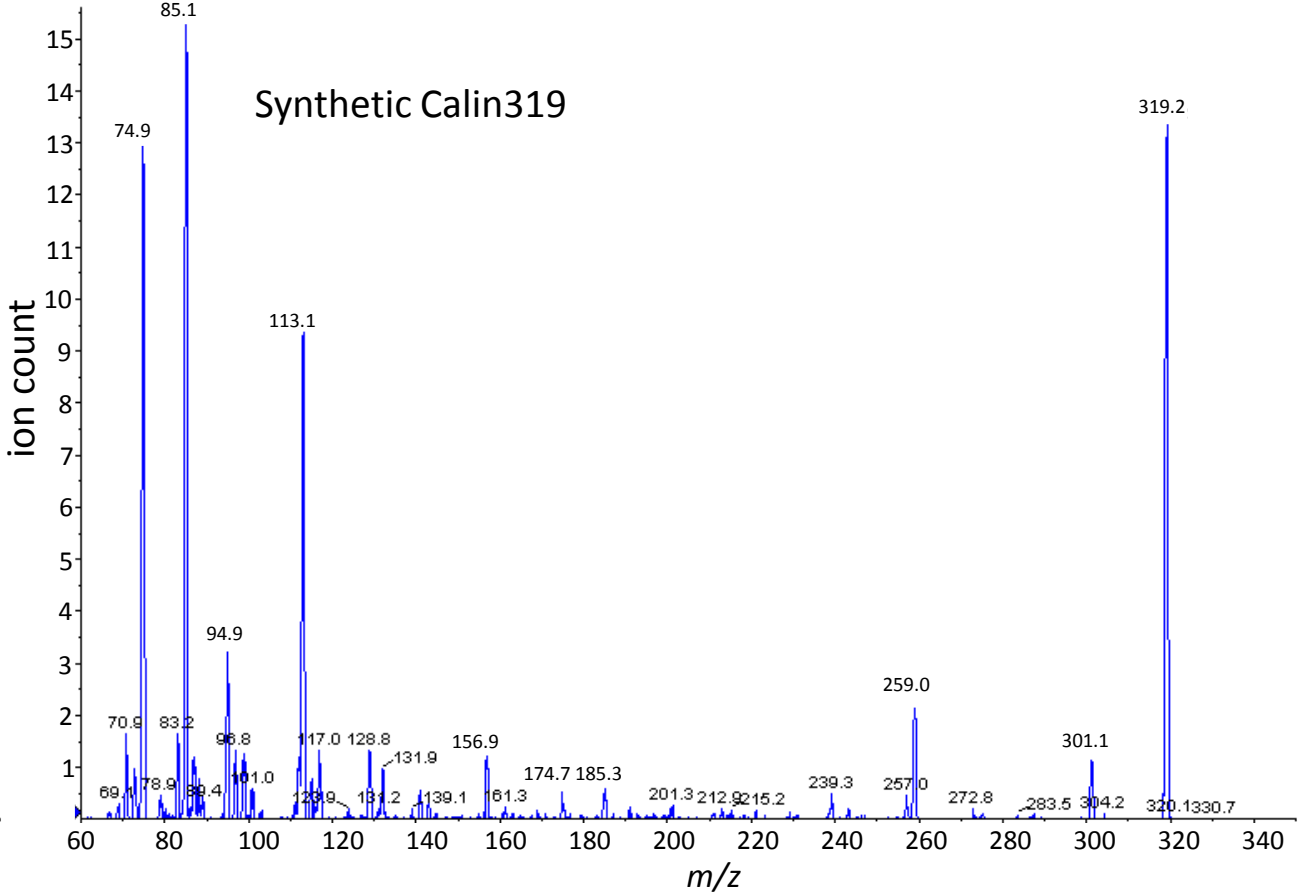

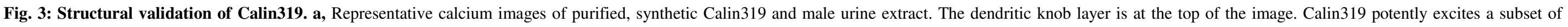

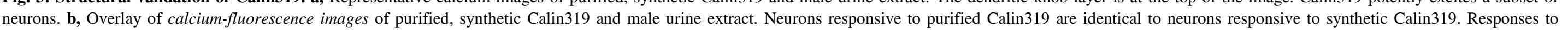

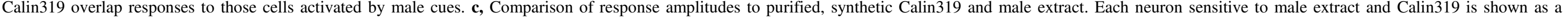

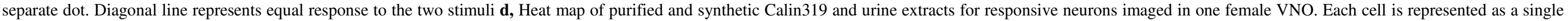

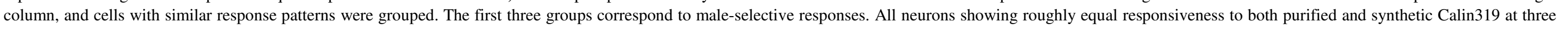

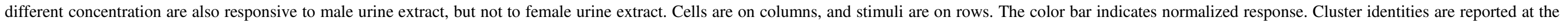
top. e, Proposed chemical structure of Calin319 (left endogenous Calin319, right synthetic Calin319) and its MS $^{2}$ product-ion spectrum. 
a

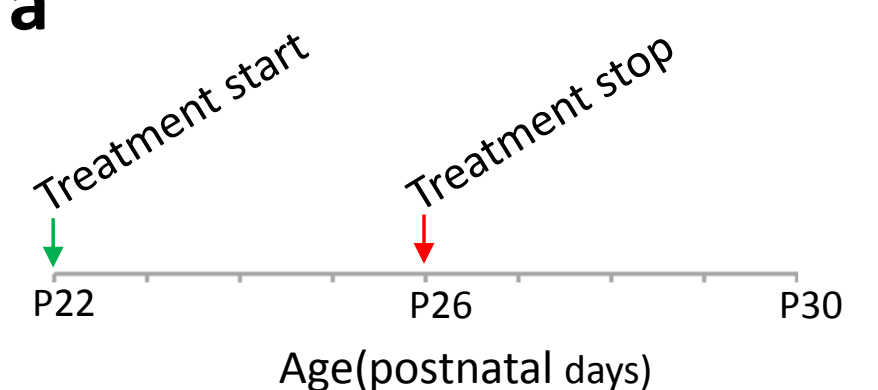

Age(postnatal days) b

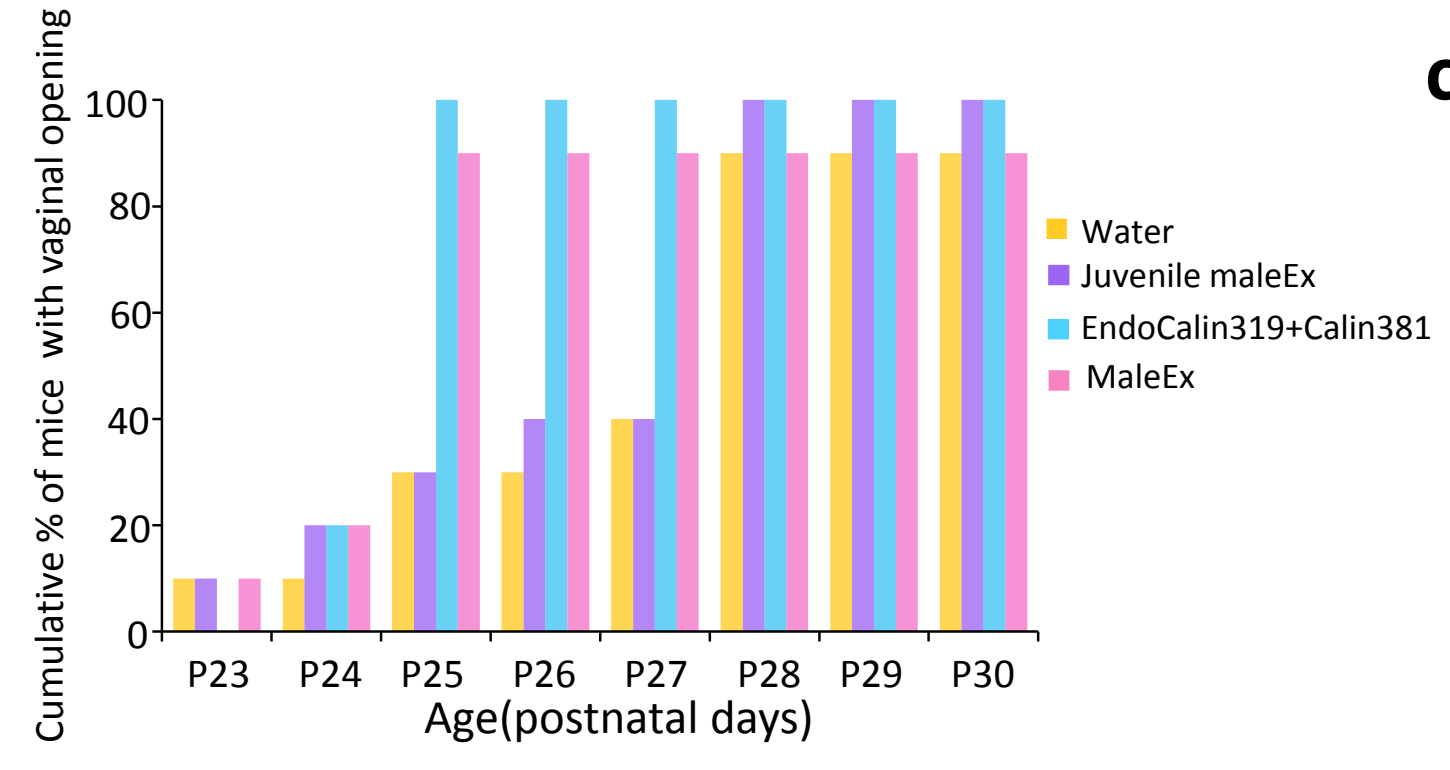

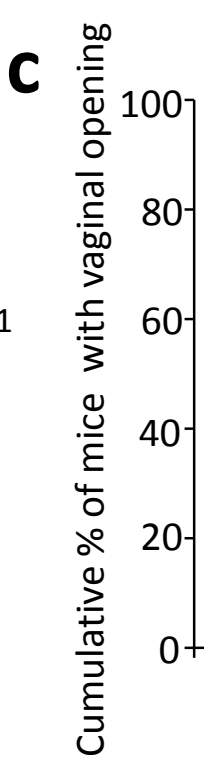

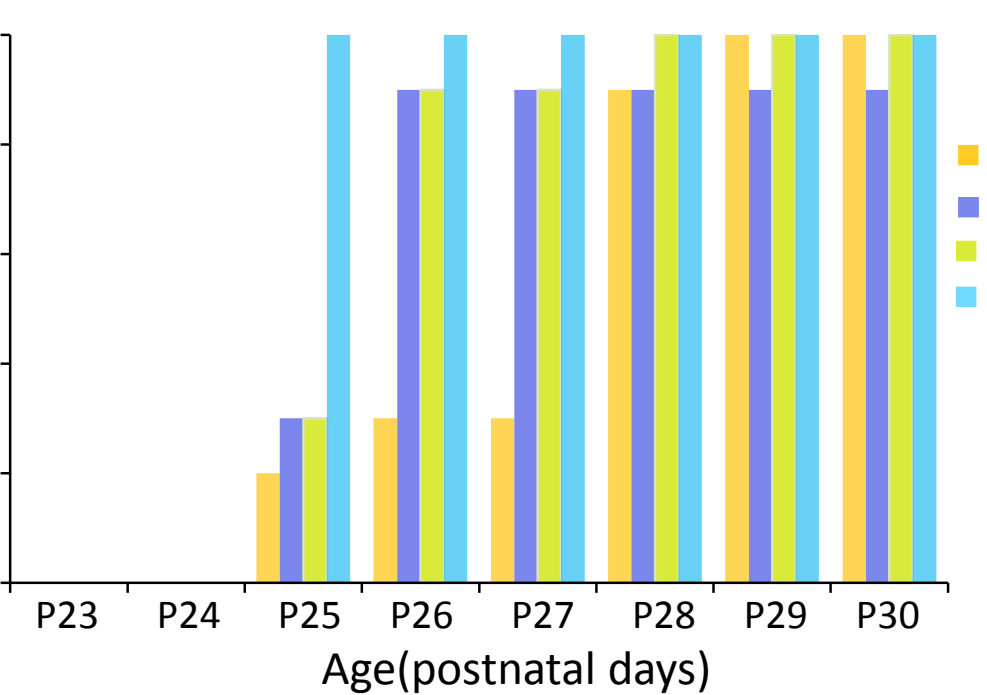

$\operatorname{TrpC2}-$
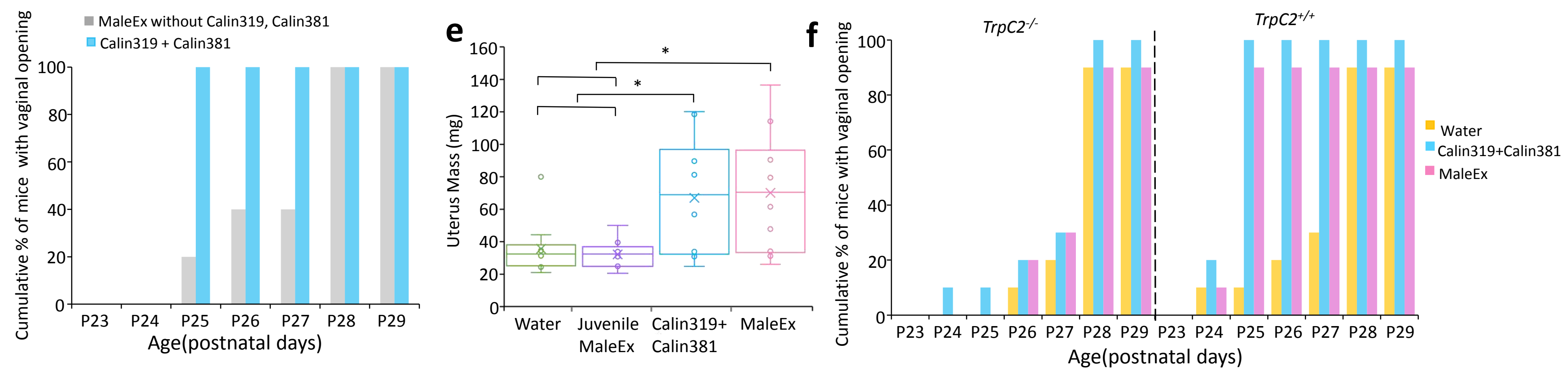

Puberty acceleration

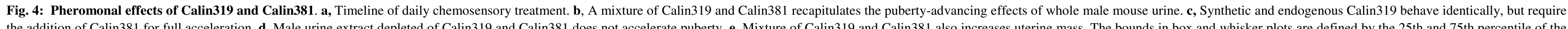

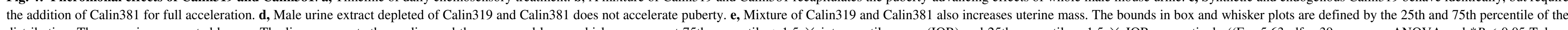

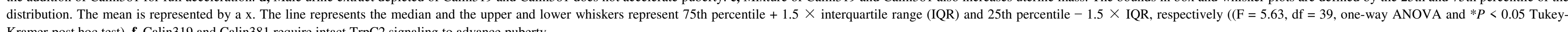
Kramer post hoc test). f, Calin319 and Calin381 require intact TrpC2 signaling to advance puberty. 
a

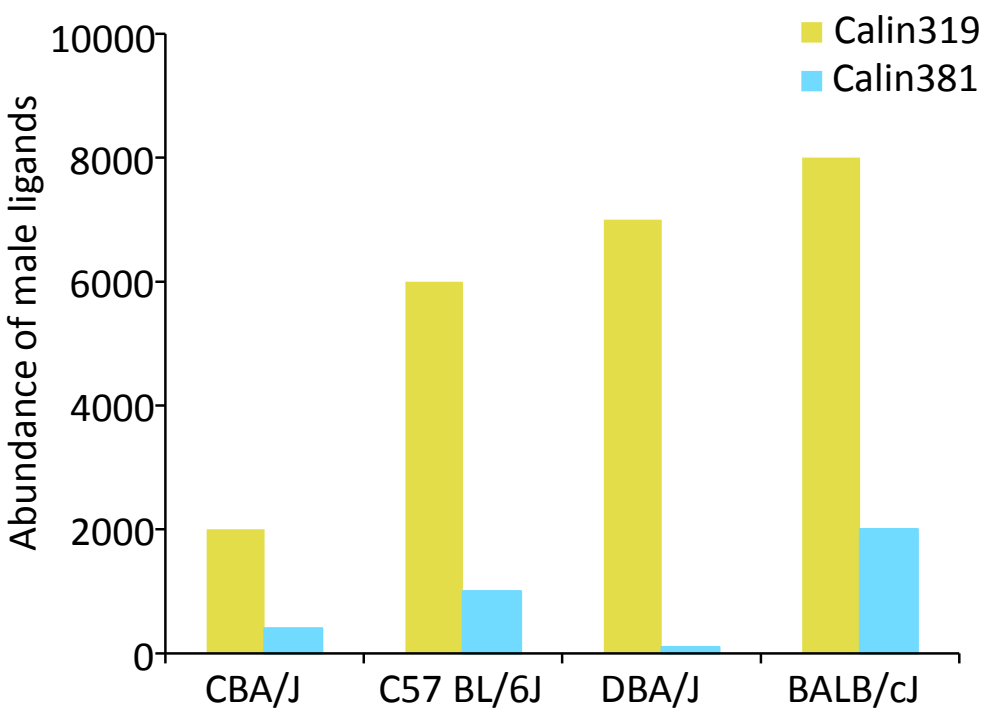

b

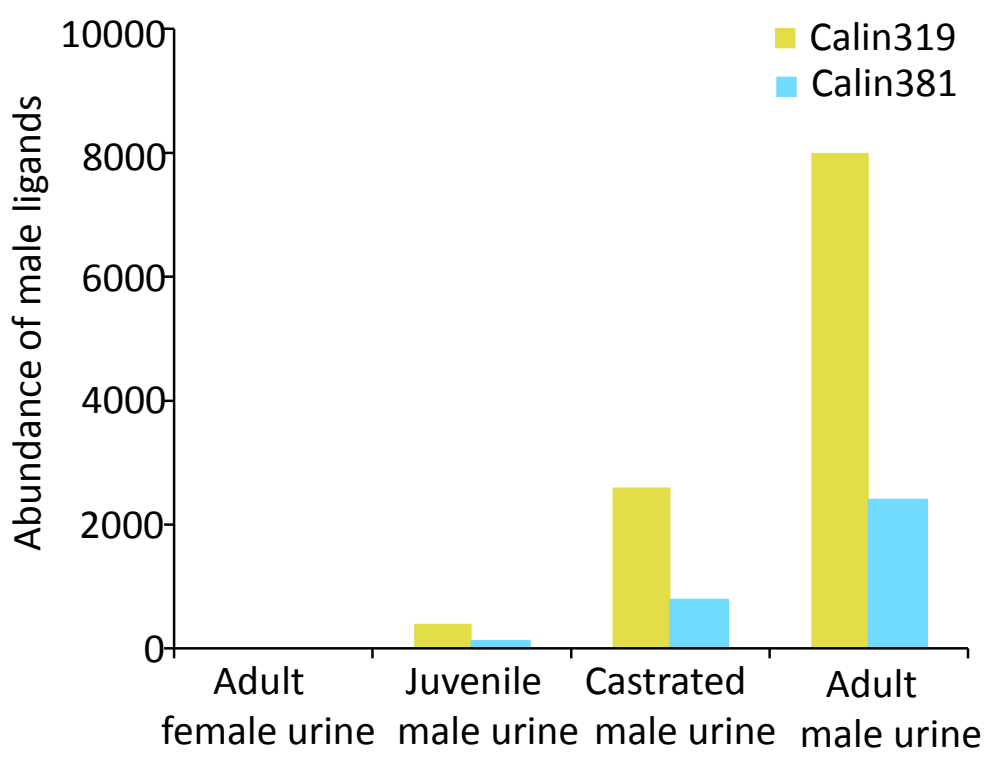

C

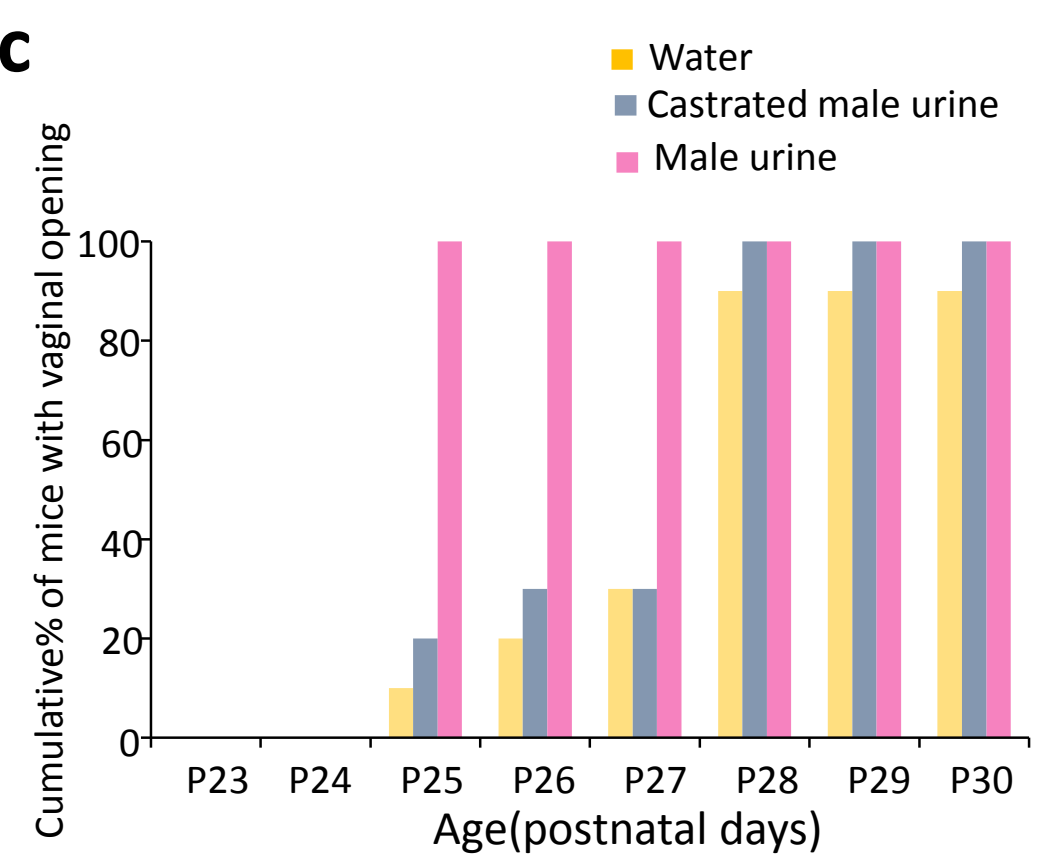

Extended data Fig. 1 Quantitative analysis of Calin319 and Calin381 across different samples and pheromonal effects of Calin319 and Calin381. a,b, Abundance of Calin319 and Calin381 across different urine samples. BALB/cJ male urine contain highest amount of Calin319 and Calin318, while they are absent from BALB/cJ female urine and scarcely detected in juvenile mouse urine. Castration significantly lowers the amount of Calin319 and Calin381. c, Puberty acceleration of different urine samples. Castrated male urine do not accelerate female puberty $(n=10$ females per group) 


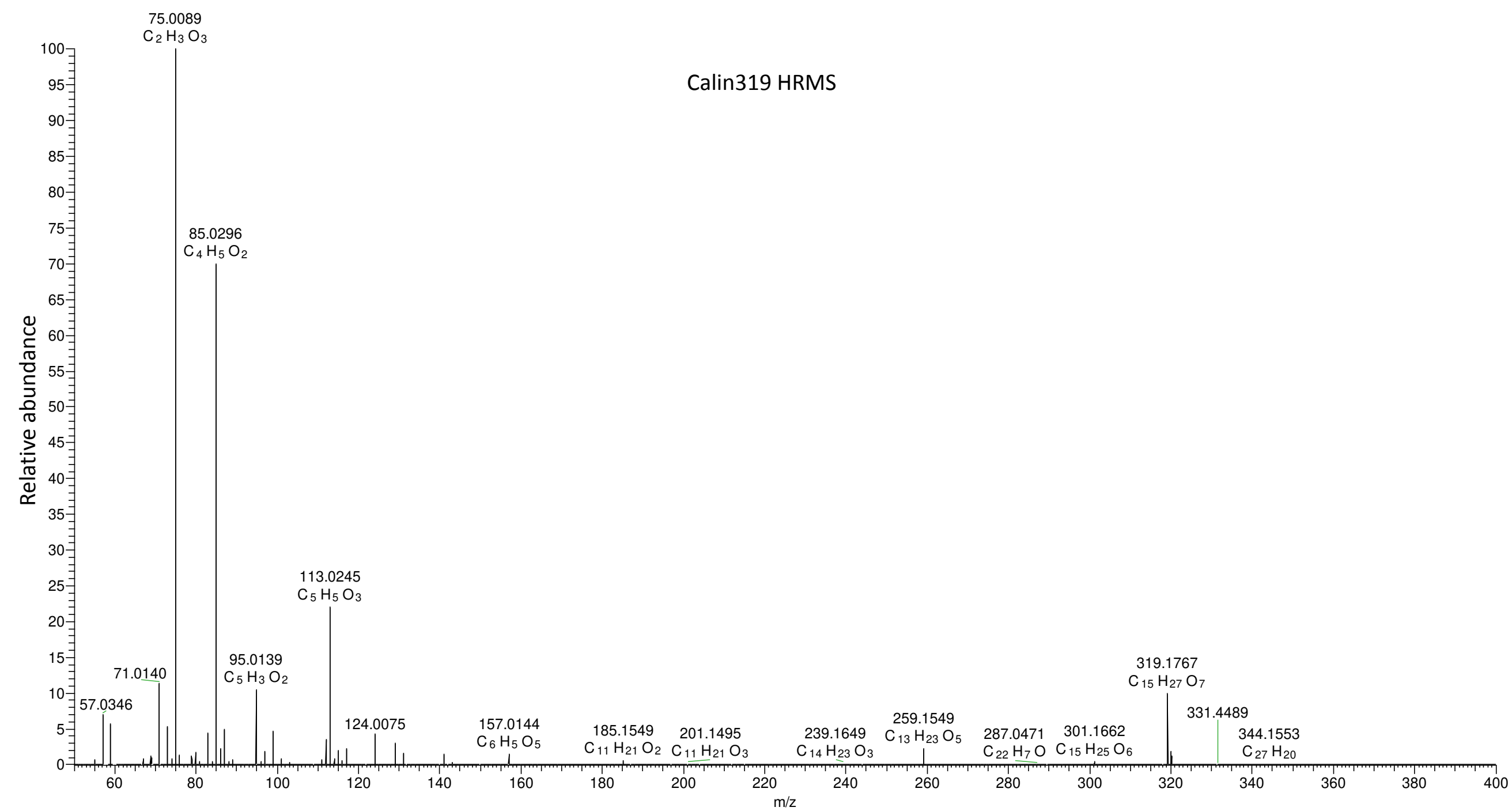

Extended data Fig. 2 High-resolution mass spectra (HRMS) of the product ion of Calin319 in the negative ion mode. 


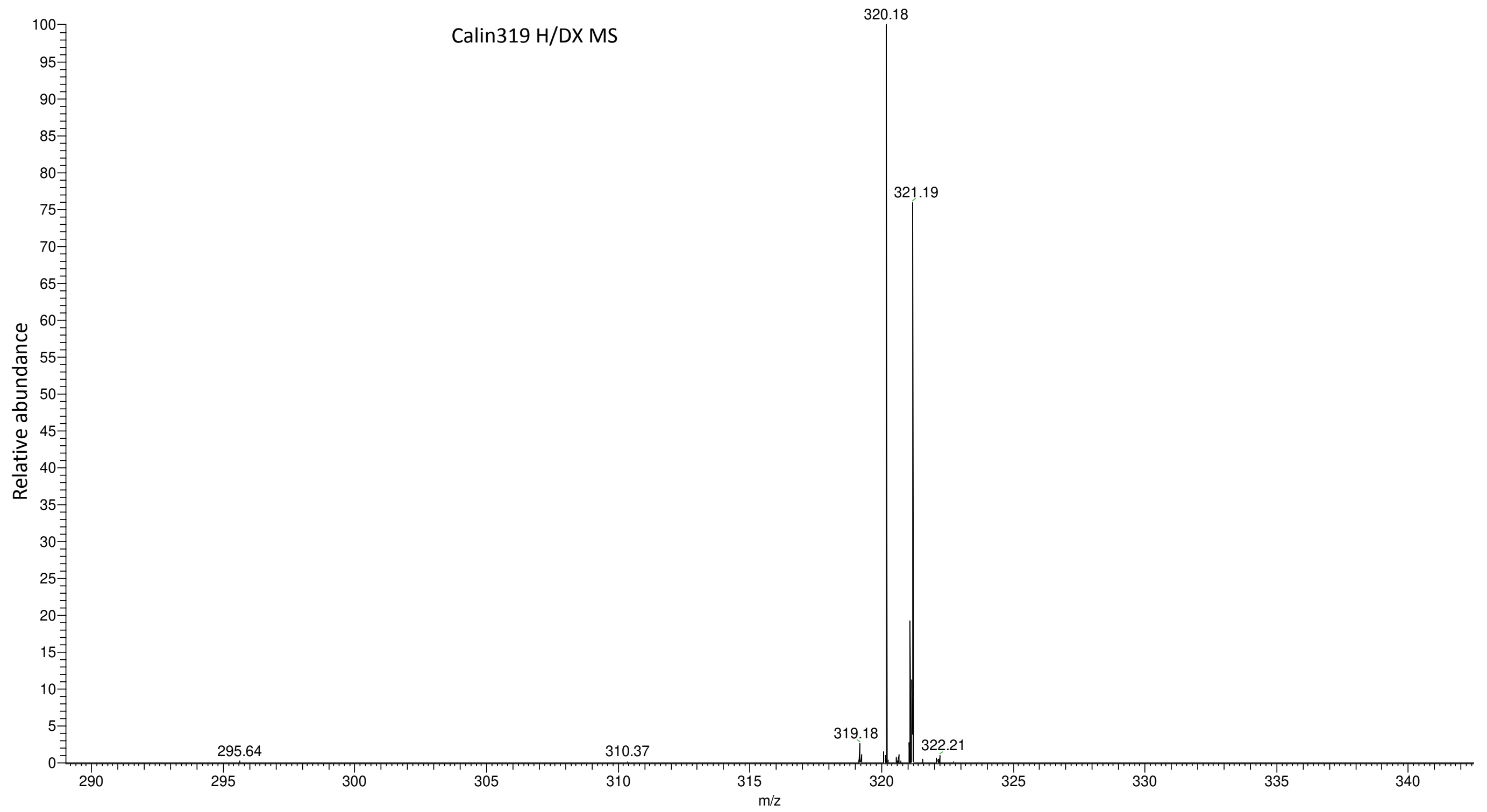

Extended data Fig. 3 Negative ion mode for Calin319 after Hydrogen/Deuterium Exchange (H/DX). 


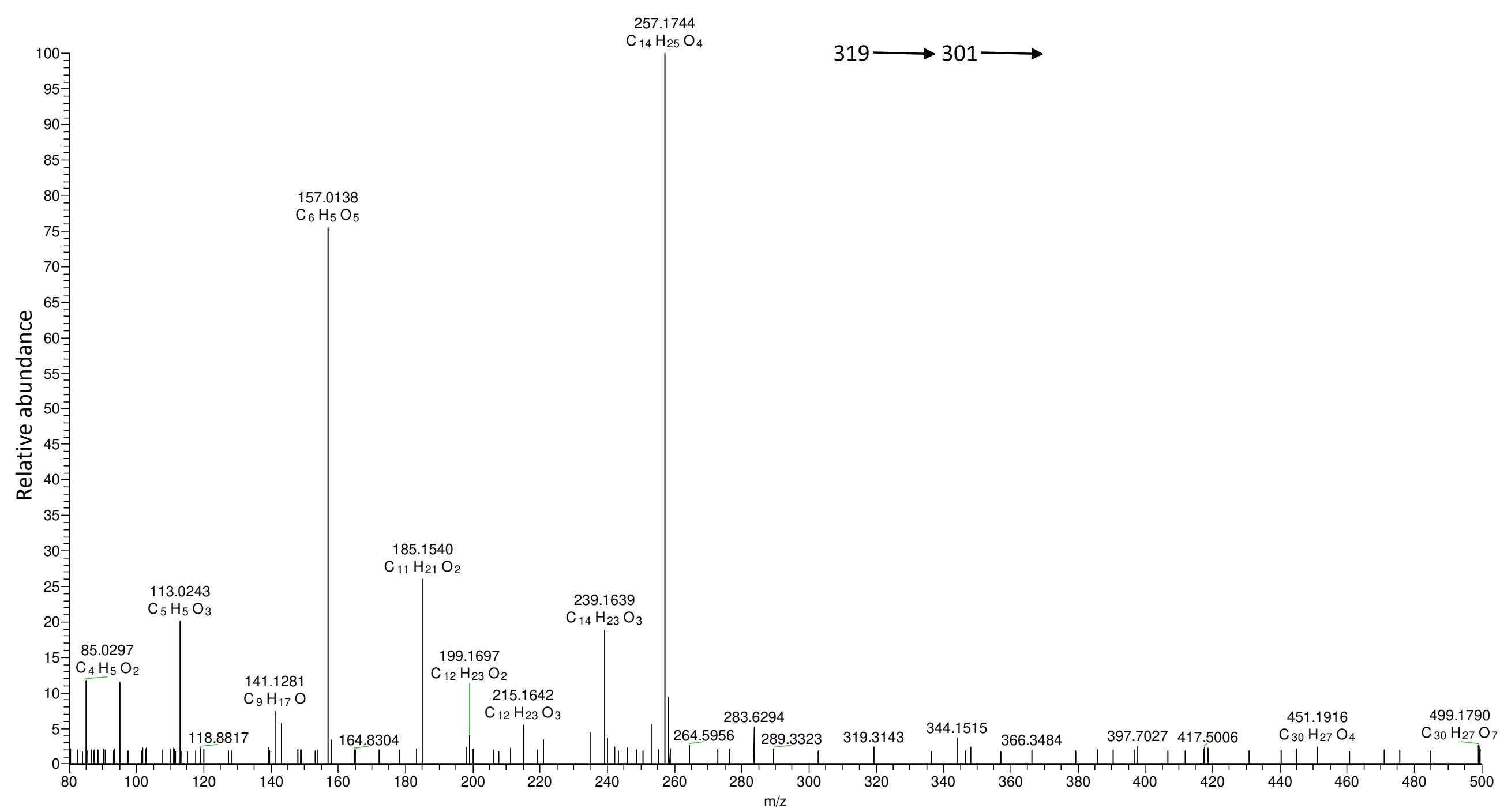

Extended data Fig. $4 \mathrm{MS}^{3}$ analysis of the product ion at $\mathrm{m} / \mathrm{z} 301$ from Calin319 $\mathrm{MS}^{2}$. 


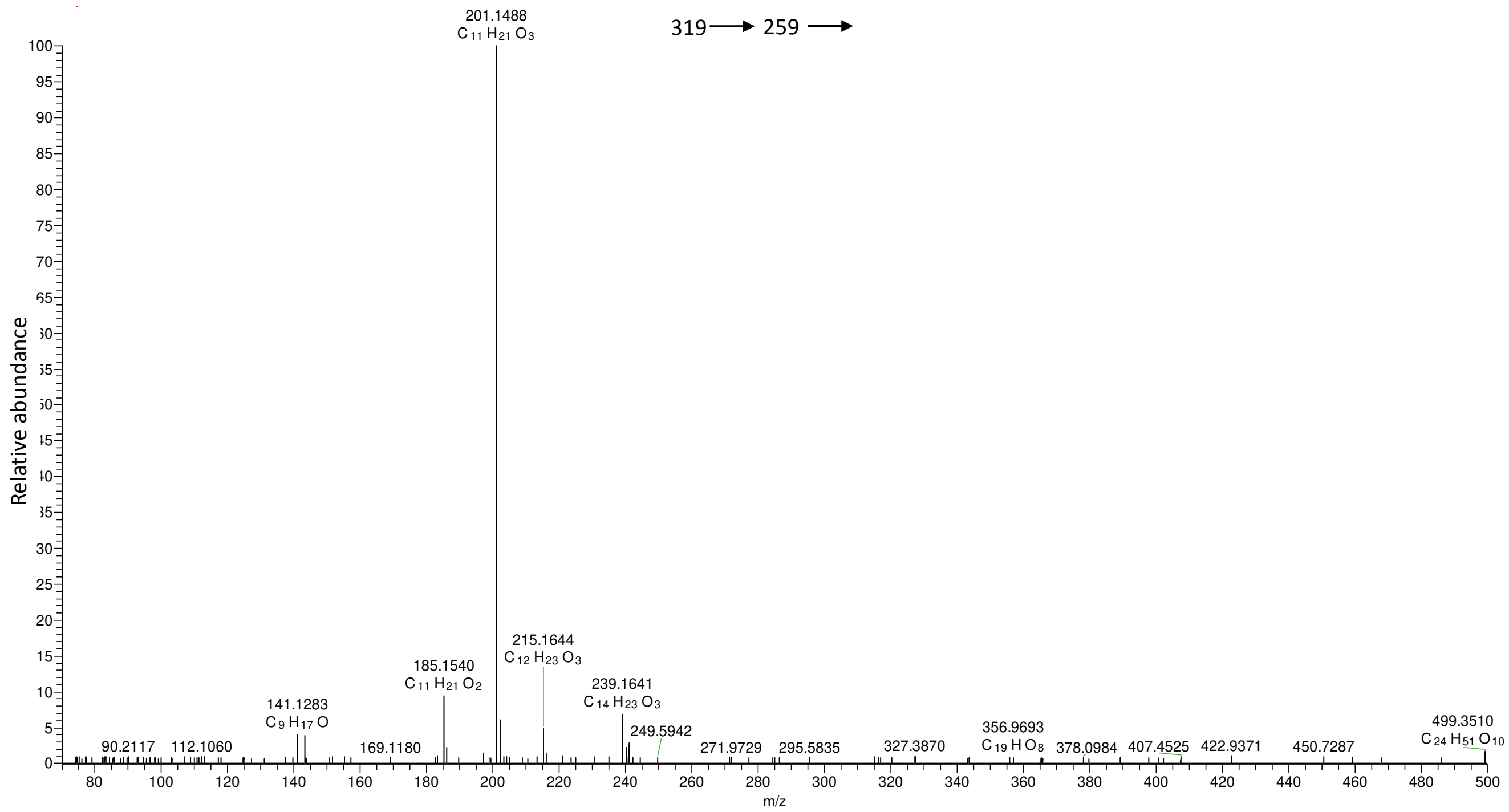

Extended data Fig. $5 \mathrm{MS}^{3}$ analysis of the product ion at $\mathrm{m} / \mathrm{z} 259$ from Calin319 $\mathrm{MS}^{2}$. 
a $N$

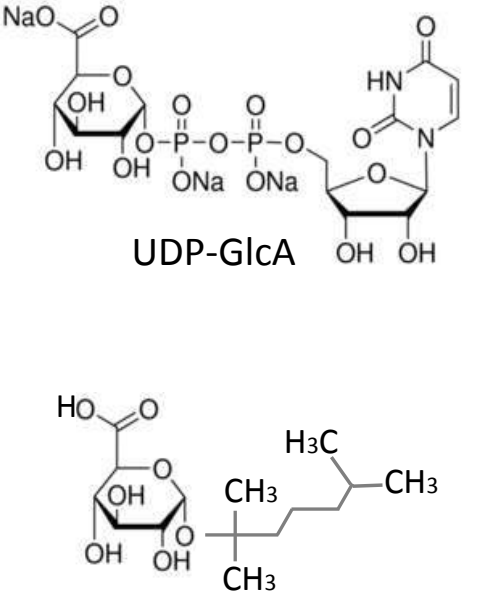

2,6-dimethyl-2-heptyl glucuronide

C

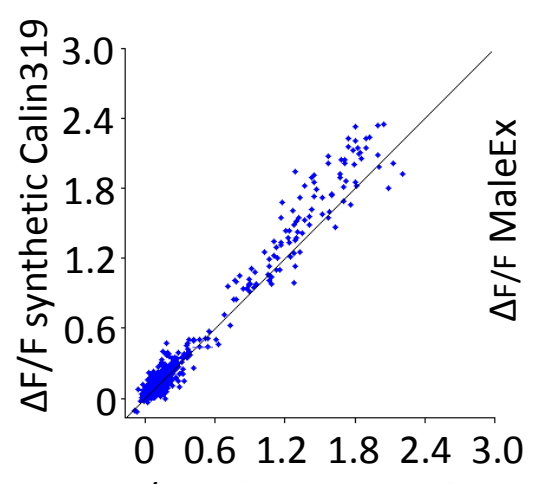

$\Delta \mathrm{F} / \mathrm{F}$ Endogenous Calin319

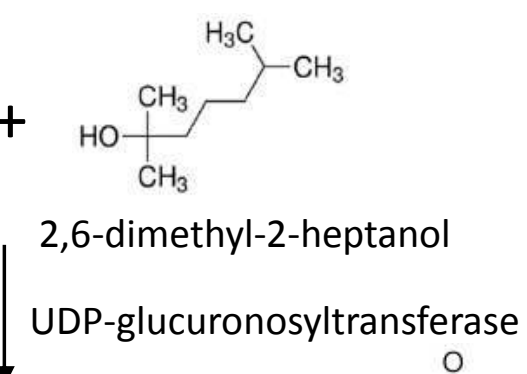

$+$

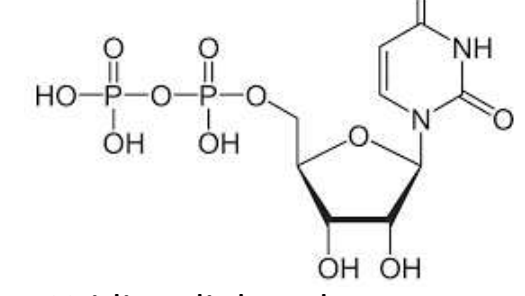

Uridine diphosphate

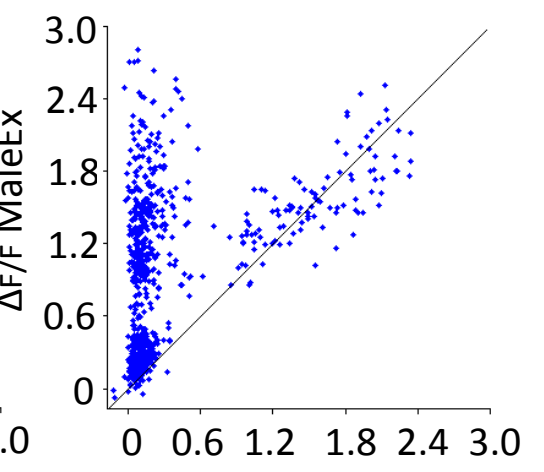

$\Delta \mathrm{F} / \mathrm{F}$ Endogenous Calin319

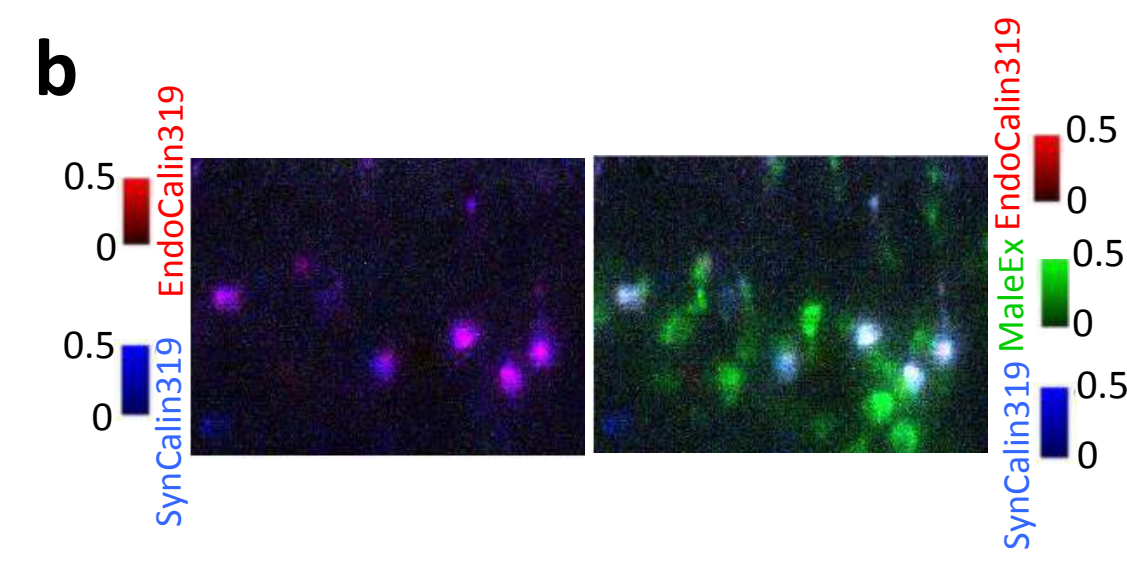

d
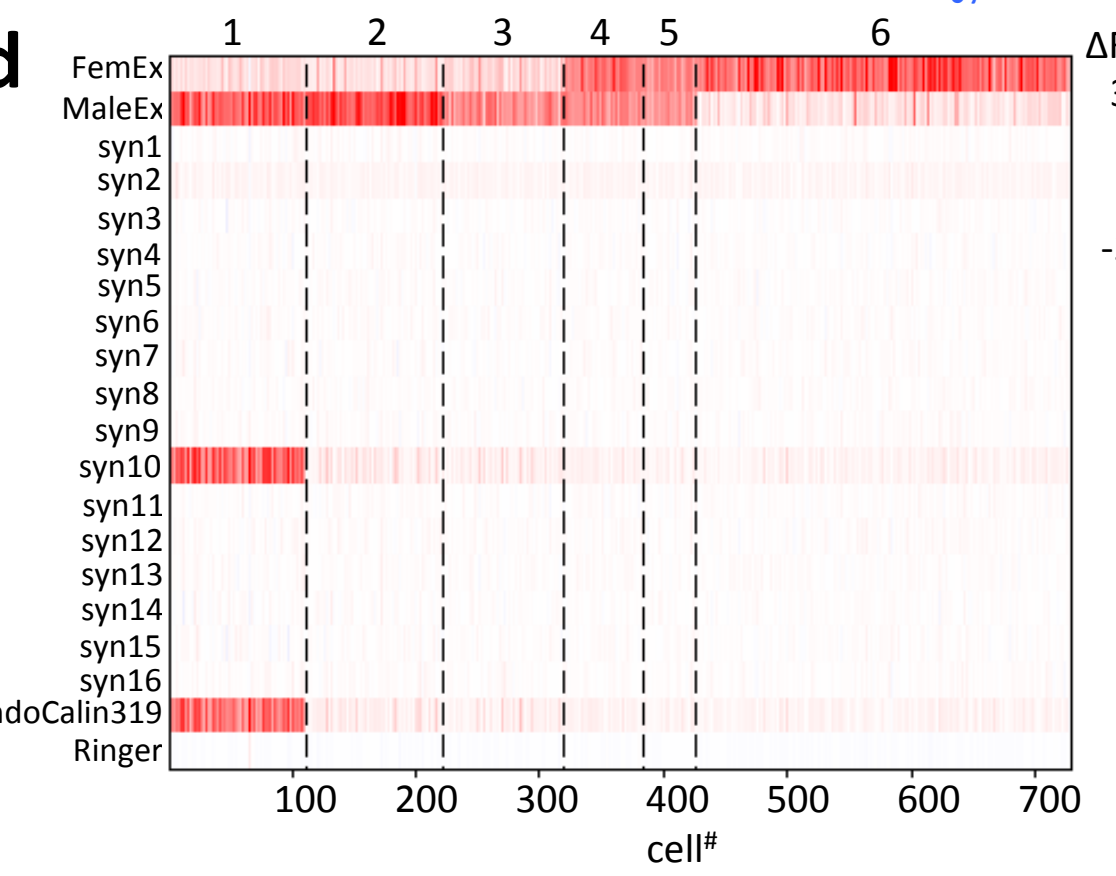

Extended data Fig. 7 Structural validation of Calin319. a, Synthesis procedure of Calin319 b, Overlay of calcium images of synthetic Calin319, endogenous Calin319 and male extract. Neurons responses to synthetic Calin319 and neurons responses to endogenous Calin319 are identical. Neuronal responses to Calin319 all overlap neuronal responses to those cells activated by male cues. c, Comparison of response amplitudes to synthetic and endogenous Calin319, endogenous Calin319 and male urine extract. Each neuron response to Calin319 and urine extract is shown as a separate dot. Diagonal line represents equal response to the two stimuli. d, Neuronal responses to Calin319 and urine extracts. Among 16 synthetic Calin319 isomers, VSNs show responses to just one synthetic Calin319 isomer. All neurons showing equal responsiveness to synthetic Calin319 and endogenous Calin319 are also responsive to male urine extract, not female urine extract. Cells are on columns, and stimuli are on rows. The color bar indicates normalized response. Cluster identities are reported at the top. 


\section{Isomers of Nonanol}
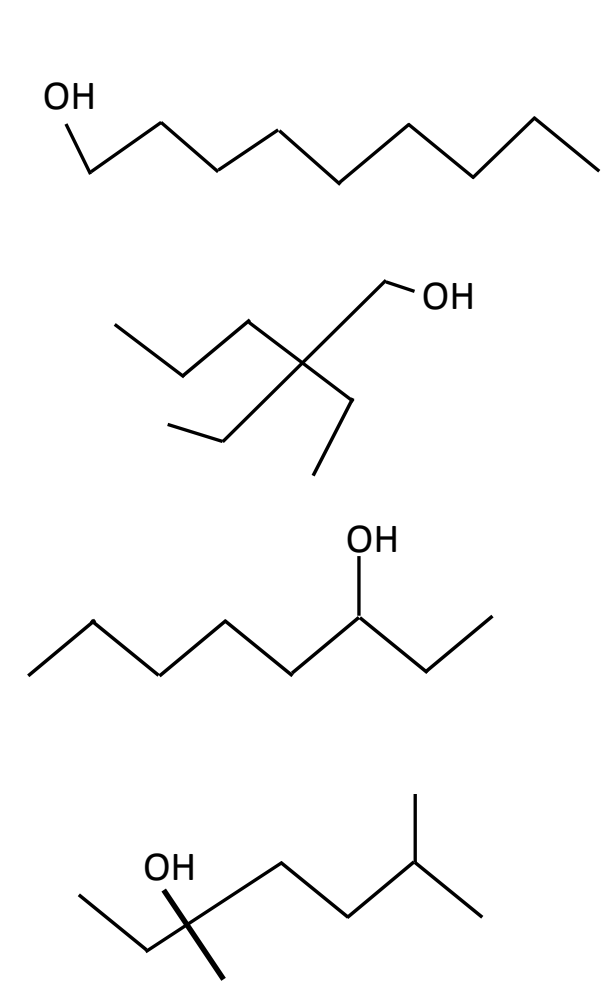<smiles>CCCCCCC(O)CCCCCCCCC(C)O</smiles><smiles>CC(CCO)CC(C)(C)C</smiles><smiles>CCCCCC(O)CCC</smiles><smiles>CCCCC(O)CC(C)C</smiles><smiles>CCCCC(O)C(C)(C)C</smiles><smiles>CCCCC(O)CCCC</smiles><smiles>CCC(C)(C)CCCCO</smiles>

Extended data Fig. 8 Isomers of nonanol tested in Extended data Fig. 7d. 


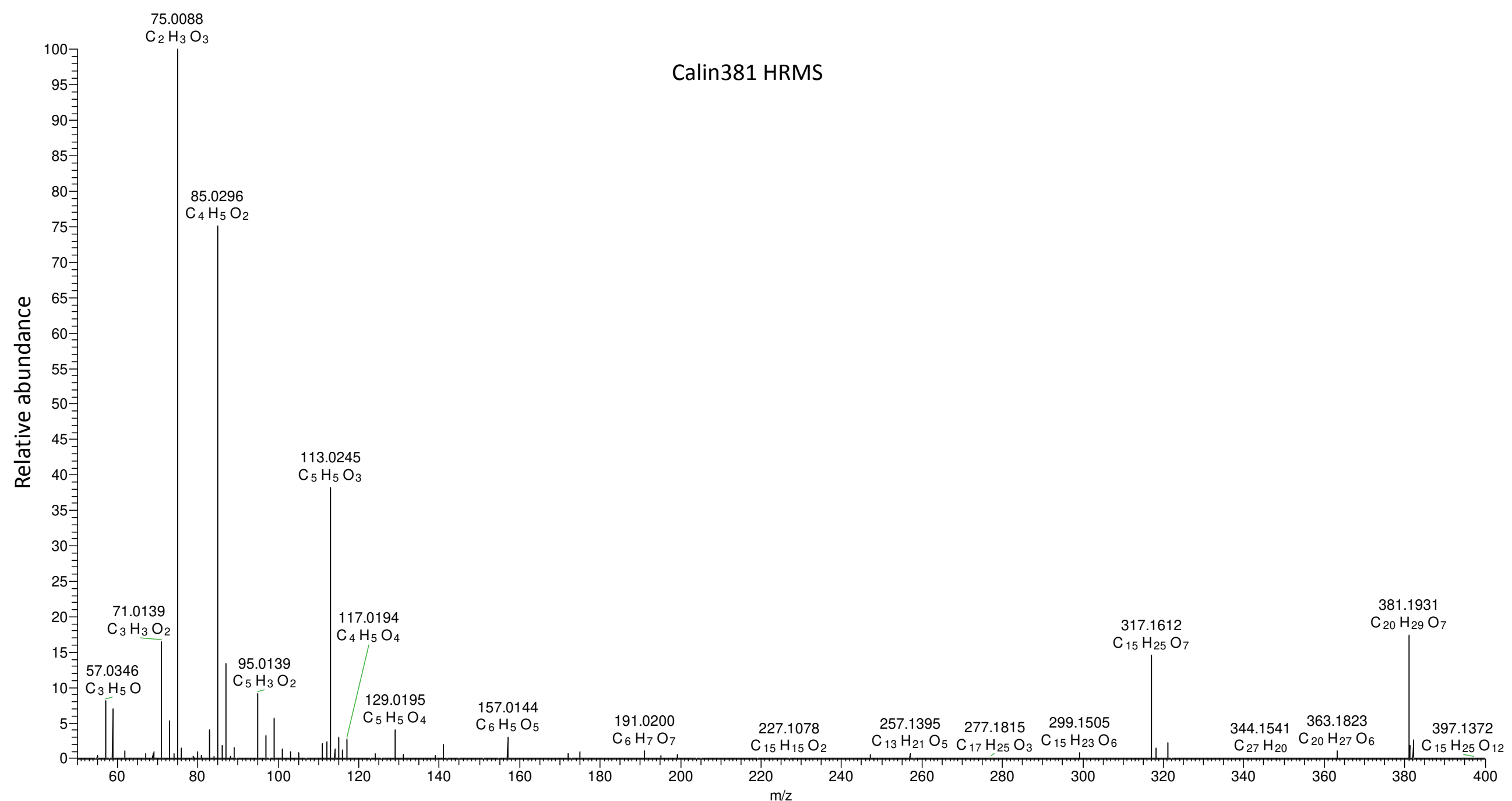

Extended data Fig. 9 High-resolution mass spectra (HRMS) of the product ion of Calin381 in the negative ion mode. 
a

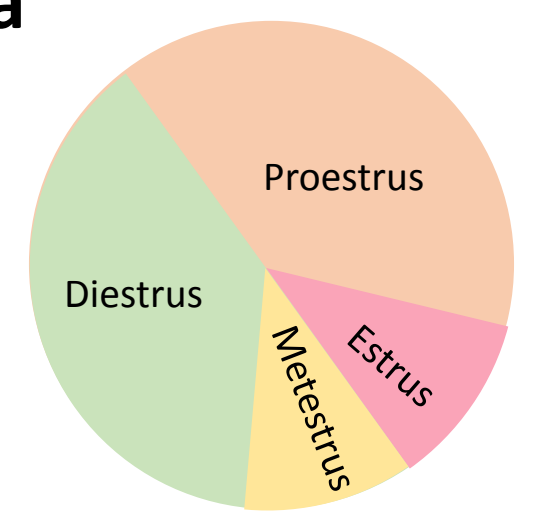

Normal estrous cycle stage

b

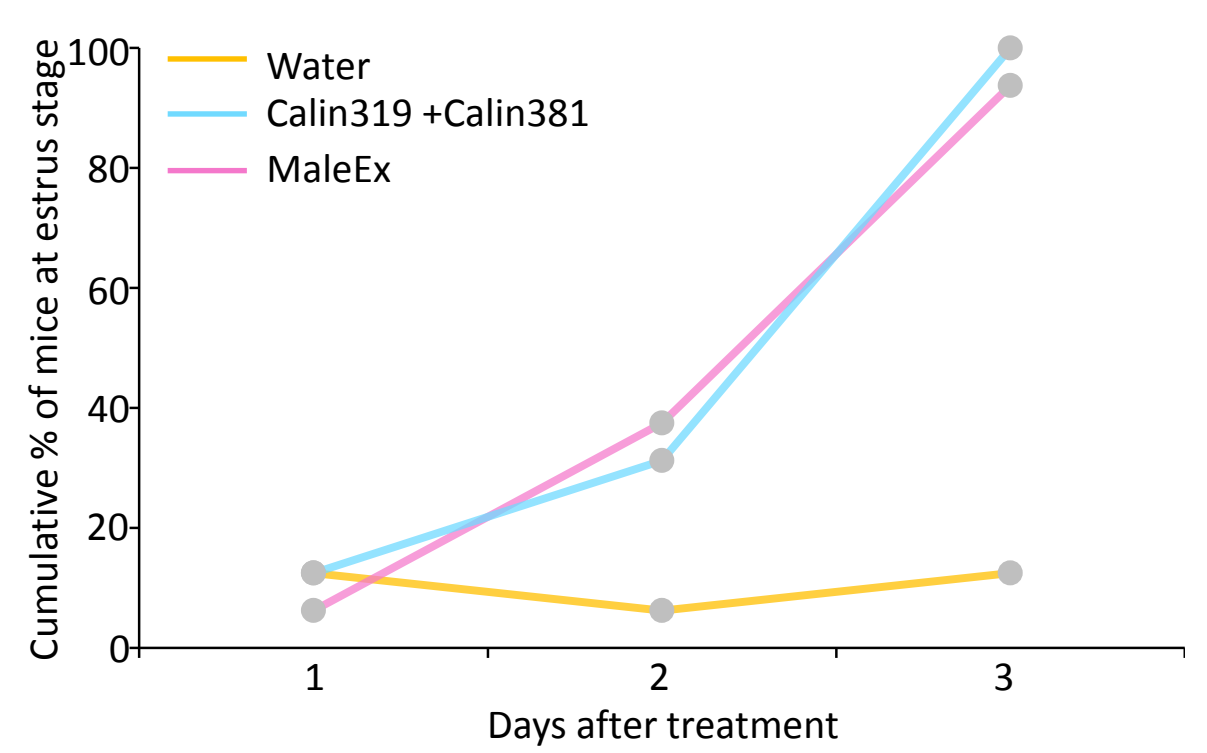

Extended data Fig. 10 Male ligands induce female estrus. a, Schematic of the puberty induction bioassay. b, A mixture of Calin319 and Calin381 triggers estrus-synchronizing effect identically to that by whole male mouse urine extract ( $n=16$ females per group).

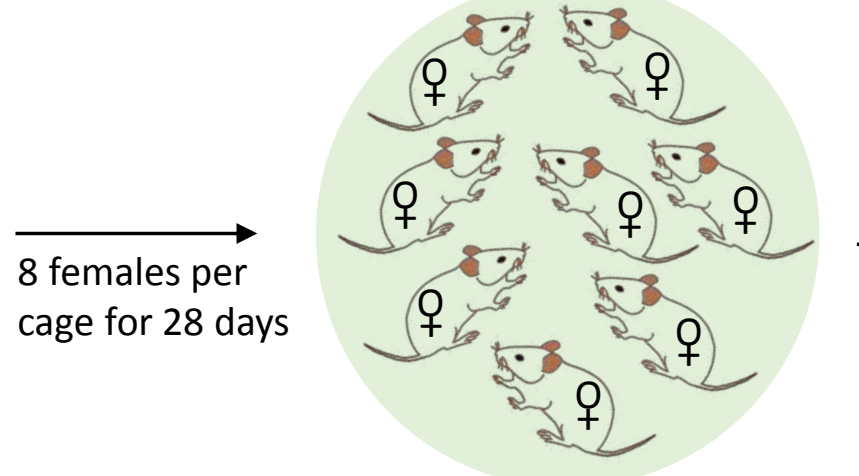

Anestrus stage

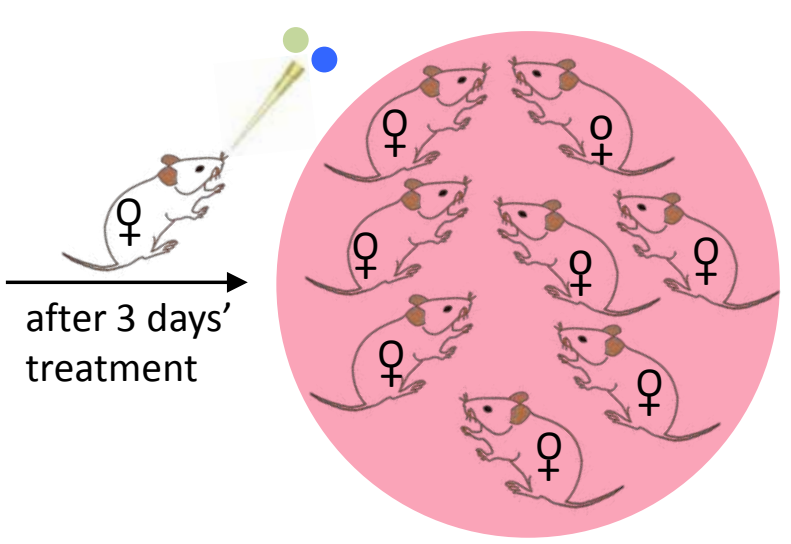

Estrus stage

Male ligands 
a

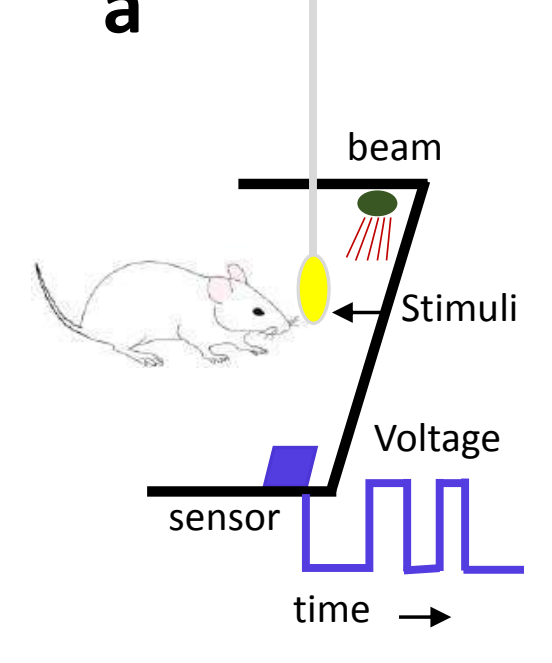

b

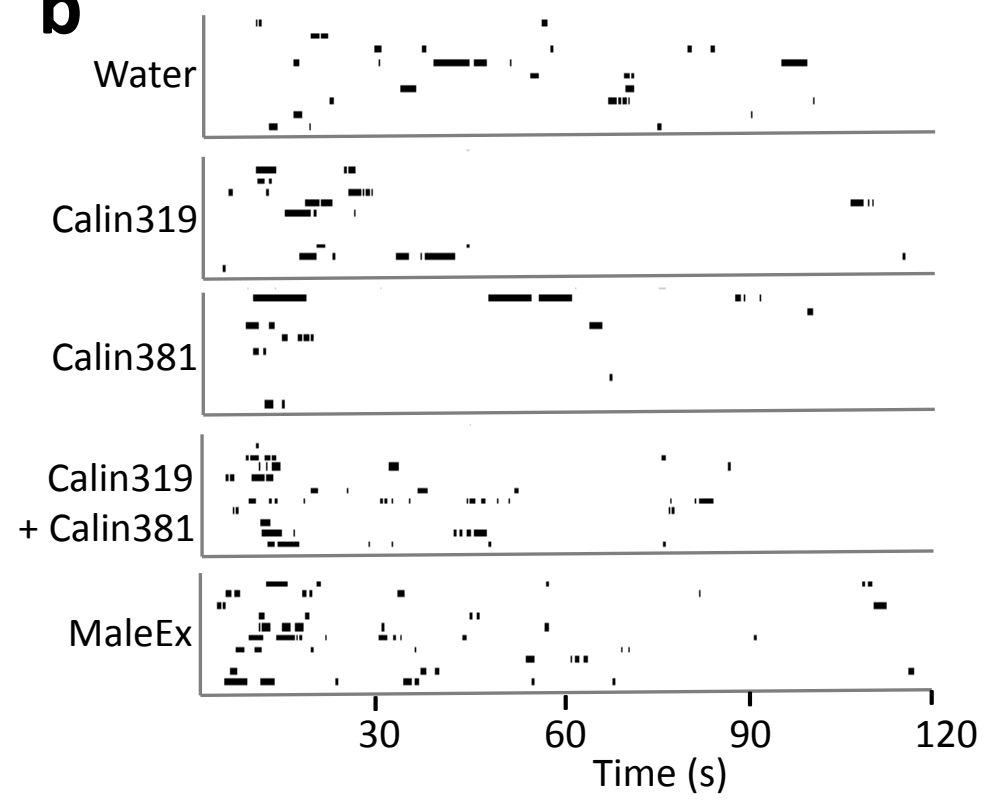

C

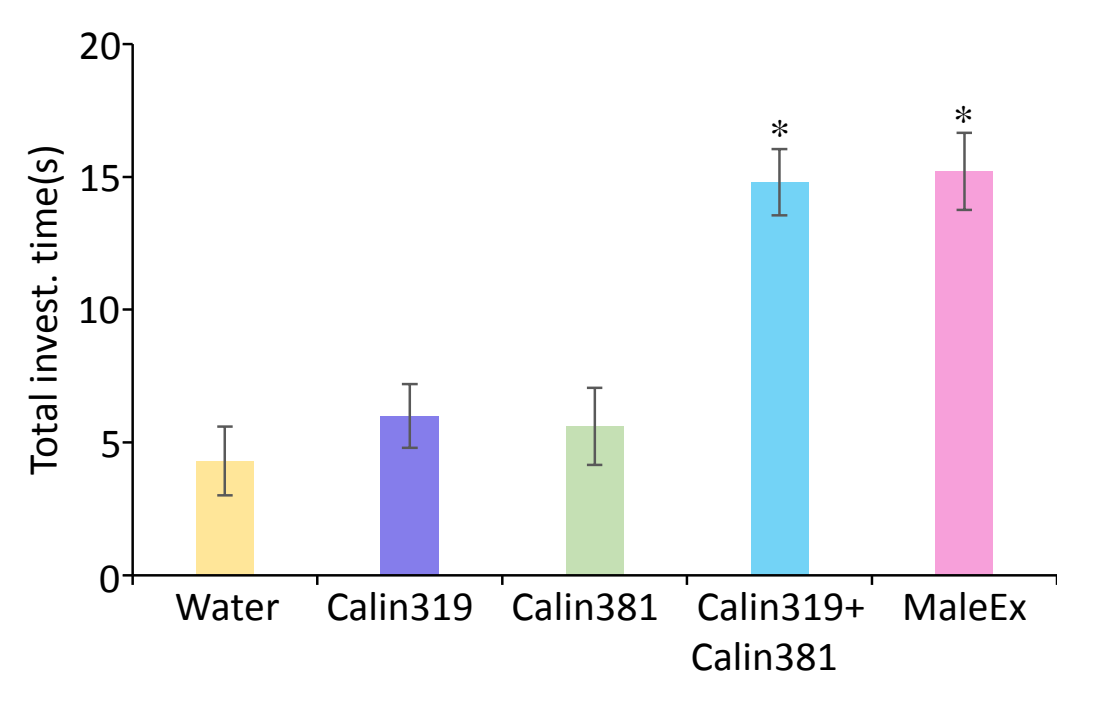

Extended data Fig. 11 Female investigator behavior to Calin319 and Calin381. a, Schematic of female investigation behavior assay. Proximal investigation time of stimuli presented on a cotton swab is measured by an optical beam-break detector. b, Episodes of beam-breaking (black bars), with each mouse displayed on a separate row. The detector and scented swab were inserted at the beginning of the trial. c, Mean and SEM of the fraction of time spent investigating the swab after initial contact. Females spent longer investigating the sample doped with a mixture of Calin319 and Calin381, and male urine extract $\left({ }^{*} p<0.05, n=10, t\right.$-test, $)$. 